\title{
THE ORIGIN OF THE HOT GAS IN THE GALACTIC HALO: CONFRONTING MODELS WITH XMM-NEWTON OBSERVATIONS
}

\author{
David B. Henley ${ }^{1}$, Robin L. Shelton ${ }^{1}$, Kyujin KwaK ${ }^{1}$, M. Ryan Joung ${ }^{2,3}$, and Mordecai-Mark Mac Low ${ }^{3}$ \\ ${ }^{1}$ Department of Physics and Astronomy, University of Georgia, Athens, GA 30602, USA; dbh@ physast.uga.edu \\ ${ }^{2}$ Department of Astronomy, Columbia University, 550 West 120th Street, New York, NY 10027, USA \\ ${ }^{3}$ Department of Astrophysics, American Museum of Natural History, 79th Street at Central Park West, New York, NY 10024, USA \\ Received 2010 May 6; accepted 2010 September 6; published 2010 October 15
}

\begin{abstract}
We compare the predictions of three physical models for the origin of the hot halo gas with the observed halo X-ray emission, derived from 26 high-latitude XMM-Newton observations of the soft X-ray background between $l=120^{\circ}$ and $l=240^{\circ}$. These observations were chosen from a much larger set of observations as they are expected to be the least contaminated by solar wind charge exchange emission. We characterize the halo emission in the XMM-Newton band with a single-temperature plasma model. We find that the observed halo temperature is fairly constant across the sky $\left(\sim(1.8-2.4) \times 10^{6} \mathrm{~K}\right)$, whereas the halo emission measure varies by an order of magnitude $\left(\sim 0.0005-0.006 \mathrm{~cm}^{-6} \mathrm{pc}\right)$. When we compare our observations with the model predictions, we find that most of the hot gas observed with XMM-Newton does not reside in isolated extraplanar supernova (SN) remnants-this model predicts emission an order of magnitude too faint. A model of an SNdriven interstellar medium, including the flow of hot gas from the disk into the halo in a galactic fountain, gives good agreement with the observed $0.4-2.0 \mathrm{keV}$ surface brightness. This model overpredicts the halo X-ray temperature by a factor of $\sim 2$, but there are a several possible explanations for this discrepancy. We therefore conclude that a major (possibly dominant) contributor to the halo X-ray emission observed with XMM-Newton is a fountain of hot gas driven into the halo by disk SNe. However, we cannot rule out the possibility that the extended hot halo of accreted material predicted by disk galaxy formation models also contributes to the emission.
\end{abstract}

Key words: Galaxy: halo - ISM: structure - X-rays: diffuse background - X-rays: ISM

Online-only material: color figure

\section{INTRODUCTION}

Observations of the diffuse soft X-ray background (SXRB) indicate the presence of $\sim(1-3) \times 10^{6} \mathrm{~K} \mathrm{X}$-ray-emitting gas in the interstellar medium (ISM) of our Galaxy. Early observations with rocket-borne instruments led to the conclusion that the diffuse $1 / 4 \mathrm{keV}$ emission was dominated by emission from $\sim 1 \times 10^{6} \mathrm{~K}$ plasma in the Local Bubble (LB), a $\sim 100$ pc cavity in the ISM in which the solar system resides (Sanders et al. 1977; Snowden et al. 1990). The discovery of shadows in the $1 / 4 \mathrm{keV}$ background with ROSAT showed that there was also gas with $T \sim 1 \times 10^{6} \mathrm{~K}$ beyond the Galactic disk, in the Galactic halo (Burrows \& Mendenhall 1991; Snowden et al. 1991). Higherenergy emission data from ROSAT, XMM-Newton, and Suzaku, and X-ray absorption line data from Chandra, show the presence of hotter gas in the Galactic halo, with temperatures up to $\sim 3 \times 10^{6}$ K (Kuntz \& Snowden 2000; Yao \& Wang 2005, 2007; Smith et al. 2007; Galeazzi et al. 2007; Henley \& Shelton 2008; Yao et al. 2009; Lei et al. 2009; Yoshino et al. 2009).

Several possible sources for the hot halo gas have been suggested, including supernova (SN)- and stellar wind-driven outflows from the Galactic disk (e.g., Shapiro \& Field 1976; Bregman 1980; Norman \& Ikeuchi 1989), gravitational heating of infalling intergalactic material (predicted by simulations of disk galaxy formation; Toft et al. 2002; Rasmussen et al. 2009), and in situ heating by extraplanar SNe (Shelton 2006; Henley \& Shelton 2009). X-ray spectroscopy is essential for determining which process or processes have produced the $\sim(1-3) \times 10^{6} \mathrm{~K}$ gas in the halo. In principle, the observed ionization state could be used to distinguish between the different models. For example, gas heated by $\mathrm{SNe}$ could be underionized if heated recently, or overionized if heated in the distant past (e.g., Shelton 1999), and gas that has recently burst out of the disk, cooling rapidly, will be drastically overionized (Breitschwerdt \& Schmutzler 1994). The elemental abundance ratios could also, in principle, be used to distinguish between models, as the abundance pattern of the hot gas may depend on whether it is of Galactic or extragalactic origin.

In practice, it is not easy to use arguments based on the ionization state or the abundances to distinguish between models, as collisional ionization equilibrium (CIE) models with solar abundances generally provide good fits to the observed X-ray spectra (e.g., Galeazzi et al. 2007; Henley \& Shelton 2008; Lei et al. 2009; Yoshino et al. 2009), although supersolar [Ne/O] and $[\mathrm{Fe} / \mathrm{O}]$ abundance ratios have been reported for some sightlines (Yoshino et al. 2009; Yao et al. 2009). Here, we use a different approach. We fit CIE models to 26 XMM-Newton spectra of the SXRB, obtained from observations between $l=120^{\circ}$ and $l=240^{\circ}$ and with $|b|>30^{\circ}$. These fits yield temperatures and emission measures (EMs) for the halo. We then compare the measured distributions of these quantities to those predicted by two physical models of the hot halo gas: a model in which the hot gas is heated in situ by extraplanar $\mathrm{SNe}$ and is contained in isolated supernova remnants (SNRs; Shelton 2006), and a model of an SN-driven ISM, one feature of which is the transfer of hot gas from the disk to the halo (Joung \& Mac Low 2006). In addition, we use our observed halo parameters to estimate the $\mathrm{X}$-ray luminosity of the halo, and compare it to the predictions of disk galaxy formation models (Toft et al. 2002; Rasmussen et al. 2009; Crain et al. 2010).

Our XMM-Newton observations are a subset of those used in the survey of Henley \& Shelton (2010, hereafter Paper I), 
who measured the SXRB O VII and O VIII intensities from 590 archival XMM-Newton observations between $l=120^{\circ}$ and $l=240^{\circ}$. The observations used here were chosen because they should be less affected by solar wind charge exchange (SWCX) emission (Cravens 2000), which is a time-varying contaminant of SXRB spectra (Cravens et al. 2001; Snowden et al. 2004; Fujimoto et al. 2007; Koutroumpa et al. 2007; Kuntz \& Snowden 2008; Carter \& Sembay 2008; Henley \& Shelton 2008). Although the 26 XMM-Newton observations used here are only a small subset of the observations used in Paper I, this is a larger number of observations than has been used in previous studies of the SXRB and the hot ISM with CCD-resolution spectra

The remainder of this paper is organized as follows. In Section 2, we present the details of our observations and give an overview of the data reduction (see Paper I for more details). Section 3 contains our spectral analysis, in which we use CIE models to determine the spectrum of the halo emission. In Section 4, we compare the results of our spectral analysis with the predictions of various physical models for the origin of the hot halo gas. In particular, the disk galaxy formation model, the extraplanar SN model, and the SN-driven ISM model are presented in Sections 4.1, 4.2, and 4.3, respectively. We discuss our results in Section 5, and conclude with a summary in Section 6. Throughout we use Anders \& Grevesse (1989) abundances.

\section{OBSERVATIONS}

Our sample of observations is taken from Paper I, which presents O VII and O vIII intensities extracted from $590 \mathrm{XMM}$ Newton observations between $l=120^{\circ}$ and $l=240^{\circ}$. The observations used here were selected by applying various filters to minimize the contamination from SWCX emission. In particular, to minimize geocoronal and near-Earth heliospheric SWCX contamination, we rejected the portions of our XMMNewton data taken when the solar wind proton flux ${ }^{4}$ exceeded $2 \times 10^{8} \mathrm{~cm}^{-2} \mathrm{~s}^{-1}$, and to minimize heliospheric SWCX contamination we used only observations of high ecliptic latitudes $\left(\beta>20^{\circ}\right)$ taken during solar minimum. ${ }^{5}$ See Section 2 of Paper I for more details about SWCX and the filters we used to reduce its effects.

After applying these filters, 43 observations remained (see Section 5.3.1 of Paper I). As we are interested in the Galactic halo here, we removed 14 more observations at low Galactic latitudes $\left(|b| \leqslant 30^{\circ}\right)$. The locations of the 29 remaining observations on the sky are shown in Figure 1. Three of these observations are toward the Eridanus Enhancement, a large, Xray-bright superbubble (Burrows et al. 1993; Snowden et al. 1995). We also removed these three observations. The details of the 26 observations that remain in our sample are summarized in Table 1.

The data reduction was carried out using SAS version 7.0.0 and the XMM-Newton Extended Source Analysis Software ${ }^{7}$ (XMM-ESAS) version 2 (Snowden \& Kuntz 2007; Kuntz $\&$ Snowden 2008). We only used data from the EPIC-MOS

\footnotetext{
4 The solar wind proton flux data were obtained from OMNIWeb (http://omniweb.gsfc.nasa.gov).

5 Following Paper I, we used observations made after 00:00UT on 2005 January 1 . This date was estimated using sunspot data from the National Geophysical Data Center

(ftp://ftp.ngdc.noaa.gov/STP/SOLAR_DATA/SUNSPOT_NUMBERS/).

6 http://xmm2.esac.esa.int/sas/7.0.0/

7 http://heasarc.gsfc.nasa.gov/docs/xmm/xmmhp_xmmesas.html
}

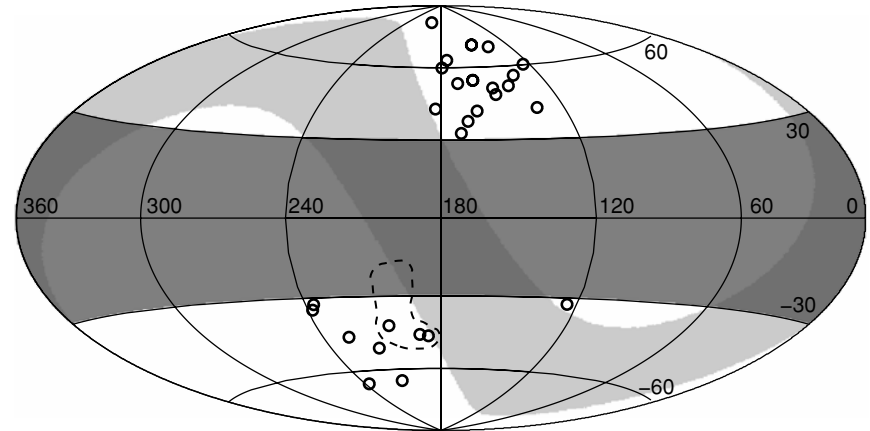

Figure 1. All-sky Hammer-Aitoff projection in Galactic coordinates, centered on the Galactic Anticenter, showing the XMM-Newton pointing directions used in this study. The gray bands indicate regions of the sky that were excluded from our sample - the darker band shows $|b| \leqslant 30^{\circ}$, and the lighter band shows $|\beta| \leqslant 20^{\circ}$. The dashed line outlines the Eridanus Enhancement. The three observations toward this feature were also excluded.

cameras (Turner et al. 2001), as the version of XMM-ESAS that we used cannot calculate the particle background for EPICpn data (Strüder et al. 2001). The data reduction method is described in full in Paper I. Here, we outline the main steps.

We cleaned and filtered each data set using the XMM-ESAS mos-filter script. This script runs the standard emchain processing script, and then uses the XMM-ESAS clean-rel program to identify and remove times affected by soft-proton flaring. As noted above, we also removed times when the solar wind proton flux exceeded $2 \times 10^{8} \mathrm{~cm}^{-2} \mathrm{~s}^{-1}$. The usable exposures for the two MOS cameras are shown in Columns 5 and 7 of Table 1.

We detected and removed point sources with a $0.5-2.0 \mathrm{keV}$ flux greater than $5 \times 10^{-14} \mathrm{erg} \mathrm{cm}^{-2} \mathrm{~s}^{-1}$. This is the approximate flux level to which Chen et al. (1997) removed sources when they measured the spectrum of the extragalactic background to be $10.5(E / 1 \mathrm{keV})^{-1.46}$ photons $\mathrm{cm}^{-2} \mathrm{~s}^{-1} \mathrm{sr}^{-1} \mathrm{keV}^{-1}$ (we use this model in our spectral analysis; see Section 3.1). Some observations contained sources that were too bright or too extended to be removed by the automated source removal. We removed such sources by hand, by excluding a circular region centered on each source.

We extracted SXRB spectra from the entire field of view, minus any sources that were removed, and minus any CCDs that were in window mode or that exhibited the anomalous state described by Kuntz \& Snowden (2008). The solid angles from which each spectrum was extracted are shown in Columns 6 and 8 of Table 1 . We binned the spectra such that each bin contained at least 25 counts. We created redistribution matrix files (RMFs) and ancillary response files (ARFs) using rmfgen and arfgen.

We used the XMM-ESAS xmm-back program to calculate the quiescent particle background (QPB) spectrum for each observation. The QPB spectra were constructed from a database of filter-wheel-closed data, scaled using data from the unexposed corner pixels that lie outside the field of view (see Kuntz \& Snowden 2008 for more details of the modeling of the QPB spectrum). The QPB spectra were subtracted from the corresponding SXRB spectra before we carried out our spectral analysis.

\section{SPECTRAL ANALYSIS}

\subsection{Model Description}

We analyzed the spectra from each of our 26 XMM-Newton observations using a multicomponent model of the SXRB. The 
Table 1

XMM-Newton Observation Details

\begin{tabular}{|c|c|c|c|c|c|c|c|c|}
\hline \multirow[t]{2}{*}{ No. } & \multirow{2}{*}{$\begin{array}{c}\text { ObsID } \\
\\
\text { (1) }\end{array}$} & \multirow{2}{*}{$\begin{array}{c}\text { Start Date } \\
\text { (2) }\end{array}$} & \multirow{2}{*}{$\begin{array}{c}l \\
(\mathrm{deg}) \\
(3)\end{array}$} & \multirow{2}{*}{$\begin{array}{c}b \\
\text { (deg) } \\
(4)\end{array}$} & \multicolumn{2}{|c|}{ MOS1 } & \multicolumn{2}{|c|}{ MOS2 } \\
\hline & & & & & $\begin{array}{c}\text { Exposure } \\
(\mathrm{ks}) \\
(5)\end{array}$ & $\begin{array}{c}\Omega \\
\left(\operatorname{arcmin}^{2}\right) \\
(6)\end{array}$ & $\begin{array}{c}\text { Exposure } \\
(\mathrm{ks}) \\
(7)\end{array}$ & $\begin{array}{c}\Omega \\
\left(\operatorname{arcmin}^{2}\right) \\
(8)\end{array}$ \\
\hline 1 & 0304070501 & $2005-11-08$ & 124.223 & 60.304 & 12.0 & 486 & 12.0 & 510 \\
\hline 2 & 0305290201 & $2005-07-02$ & 124.578 & -32.485 & 15.1 & 478 & 16.9 & 572 \\
\hline 3 & 0401210601 & $2006-10-10$ & 133.225 & 42.419 & 17.7 & 310 & 17.6 & 394 \\
\hline 4 & 0404220101 & 2006-11-01 & 135.974 & 55.981 & 13.0 & 488 & 14.1 & 505 \\
\hline 5 & 0400560301 & $2006-11-17$ & 138.279 & 68.853 & 51.5 & 380 & 51.5 & 403 \\
\hline 6 & 0400570201 & $2006-11-25$ & 142.370 & 51.705 & 23.0 & 463 & 22.9 & 475 \\
\hline 7 & 0406630201 & $2007-04-12$ & 151.186 & 48.245 & 8.5 & 339 & 8.4 & 431 \\
\hline 8 & 0303260201 & 2005-04-07 & 151.607 & 51.006 & 44.4 & 411 & 44.0 & 583 \\
\hline 9 & 0306060201 & $2005-11-13$ & 151.829 & 70.103 & 53.5 & 410 & 54.8 & 511 \\
\hline 10 & 0306060301 & $2005-11-15$ & 151.831 & 70.103 & 15.3 & 415 & 15.6 & 511 \\
\hline 11 & 0303720601 & $2005-04-25$ & 161.440 & 54.439 & 23.5 & 382 & 22.9 & 398 \\
\hline 12 & 0303720201 & $2005-04-13$ & 161.441 & 54.439 & 25.8 & 378 & 26.2 & 470 \\
\hline 13 & 0200960101 & $2005-03-28$ & 162.721 & 41.656 & 56.9 & 453 & 57.0 & 465 \\
\hline 14 & 0301340101 & 2006-04-12 & 167.648 & 37.517 & 12.8 & 487 & 13.0 & 512 \\
\hline 15 & 0306370601 & $2005-04-24$ & 170.477 & 53.178 & 9.6 & 496 & 10.1 & 583 \\
\hline 16 & 0402780701 & $2007-03-28$ & 171.132 & 32.731 & 14.3 & 422 & 14.6 & 577 \\
\hline 17 & 0304203401 & 2006-06-11 & 175.807 & 63.353 & 8.5 & 371 & 8.3 & 391 \\
\hline 18 & 0406610101 & $2006-11-05$ & 179.356 & 59.942 & 10.4 & 402 & 10.8 & 485 \\
\hline 19 & 0400830301 & $2006-10-30$ & 182.658 & 42.566 & 45.0 & 493 & 44.7 & 511 \\
\hline 20 & 0301651701 & 2006-06-20 & 197.309 & 81.121 & 12.3 & 473 & 12.2 & 495 \\
\hline 21 & 0300630301 & 2006-01-19 & 209.821 & -65.146 & 14.4 & 477 & 14.5 & 499 \\
\hline 22 & 0312190601 & $2006-01-28$ & 213.849 & -50.846 & 11.3 & 391 & 11.2 & 477 \\
\hline 23 & 0301330401 & $2006-02-13$ & 226.946 & -45.906 & 19.5 & 414 & 19.6 & 583 \\
\hline 24 & 0312190701 & $2006-01-28$ & 236.040 & -32.583 & 11.1 & 483 & 10.8 & 571 \\
\hline 25 & 0302500101 & 2005-08-09 & 237.074 & -65.638 & 21.9 & 413 & 23.5 & 583 \\
\hline 26 & 0307001401 & $2006-02-13$ & 237.615 & -34.679 & 7.8 & 489 & 8.3 & 584 \\
\hline
\end{tabular}

Notes. The observations are in order of increasing $l$. Column 1 contains the XMM-Newton observation ID. Column 2 contains the observation start date, in YYYY-MM-DD format. Columns 3 and 4 contain the pointing direction in Galactic coordinates. Column 5 contains the usable MOS1 exposure time that remains after the filtering mentioned in Section 2. Column 6 contains the solid angle, $\Omega$, from which the MOS1 SXRB spectrum was extracted, after the removal of sources and unusable CCDs. Columns 7 and 8 contain the corresponding data for MOS2.

model consisted of the following components: a foreground emission component (representing LB emission and/or SWCX emission that remains in our spectra, despite our efforts to minimize this contamination), a Galactic halo component, an extragalactic component, and instrumental background components.

We used a Raymond \& Smith (1977 and updates) model with $T \sim 10^{6} \mathrm{~K}$ to model the foreground emission in our analysis, because such a model provides a good fit to data from the ROSAT All-Sky Survey (e.g., Snowden et al. 1998, 2000; Kuntz \& Snowden 2000). We fixed the temperature $\left(T=1.2 \times 10^{6} \mathrm{~K}\right)$ and normalization of this component using data from the Snowden et al. (2000) catalog of shadows in the ROSAT All-Sky Survey. Following Paper I, we calculated the foreground R12 (1/4 keV) count rate for each XMM-Newton sightline by averaging the foreground count rates of the five nearest shadows, weighted by their inverse distances from the $X M M-N e w t o n$ sightline, i.e.,

$$
\text { Foreground R12 count rate }=\frac{\sum R_{i} / \theta_{i}}{\sum 1 / \theta_{i}}
$$

where $R_{i}$ is the foreground $\mathrm{R} 12$ count rate for the $i$ th shadow and $\theta_{i}$ is the angle between the center of the $i$ th shadow and the $X M M$-Newton sightline. Over the whole set of XMM-Newton sightlines, the median value of $\theta_{i}$ is 6.2 (lower and upper quartiles: 4.1 and 7.7, respectively). The median minimum and maximum values of $\theta_{i}$ for each sightline are 2.8 and 7.4 , respectively. Only three sightlines have $\theta_{i}>7^{\circ}$ for all $i$ : obs. $0305290201\left(\theta_{i}=7.4-9.3\right)$, obs. $0301340101\left(\theta_{i}=8.6-14.5\right)$, and obs. $0402780701\left(\theta_{i}=11.2-14.7\right)$. We used the abovecalculated count rate to determine the normalization of the foreground component for the sightline in question (assuming $T=1.2 \times 10^{6} \mathrm{~K}$ ). This normalization was held fixed during the subsequent spectral fitting.

For the Galactic halo, which is the component of the SXRB that we are interested in here, we used a single-temperature (1T) Raymond \& Smith (1977 and updates) model. While such a halo model is inadequate for explaining all the available Xray and far-ultraviolet data (Yao \& Wang 2007; Shelton et al. 2007; Lei et al. 2009), it is adequate for characterizing the Xray emission in the XMM-Newton band. The temperature and EM of this component were both free parameters in the spectral fitting. We used a Raymond \& Smith model, instead of, say, an APEC model, because the codes that we used to calculate X-ray spectra from hydrodynamical models (see Section 4) also use the Raymond \& Smith code. In our analysis, the temperature and EM of the Galactic halo component were free to vary.

We modeled the extragalactic background as a power law with a photon index $\Gamma=1.46$ and a normalization of 10.5 photons $\mathrm{cm}^{-2} \mathrm{~s}^{-1} \mathrm{sr}^{-1} \mathrm{keV}^{-1}$ at $1 \mathrm{keV}$ (Chen et al. 1997). The extragalactic and halo components were both subject to absorption. For each sightline, we used the HEAsoft nh tool to 
obtain the H I column density from the Leiden-Argentine-Bonn (LAB) Survey of Galactic H I (Kalberla et al. 2005). We used photoelectric absorption cross sections from Bałucińska-Church \& McCammon (1992), with an updated He cross section from Yan et al. (1998).

As well as the above SXRB components, we included components to model parts of the particle background. These components were independent for the two MOS detectors. The QPB spectrum includes two bright fluorescent lines from aluminum and silicon at 1.49 and $1.74 \mathrm{keV}$, respectively. The procedure for calculating the QPB spectrum mentioned in Section 2 cannot adequately remove these lines. Instead, the QPB spectrum was interpolated between 1.2 and $1.9 \mathrm{keV}$, and we added two Gaussians to our spectral model to model these lines. In addition, despite the data cleaning described in Section 2, some residual soft-proton contamination may remain in the data. We modeled this contamination using a power law which we did not fold through the instrumental response (Snowden \& Kuntz 2007, 2010). Following suggestions in the latest version of the XMM-ESAS manual (Snowden \& Kuntz 2010, which pertains to a later version of the software than the one we used), we placed constraints on the spectral index of this power law (specifically, soft limits at 0.5 and 1.0, and hard limits at 0.2 and 1.3).

Originally, as in Paper I, we used a broken power law to model the soft protons. We fixed the break at $3.2 \mathrm{keV}$ (Kuntz \& Snowden 2008), but we did not impose any special constraints on the spectral indices. We find that the halo temperatures are generally not significantly affected by our choice of soft-proton model, but the EMs and surface brightnesses are typically $30 \%$ higher if we place constraints on the power-law spectral index. However, these differences do not affect the conclusions of this paper. Throughout this paper, we use the newer set of spectral fit results, obtained using an unbroken power law with constraints on the spectral index to model the soft protons.

We carried out our spectral analysis using $\mathrm{XSPEC}^{8}$ version 12.5.0. We analyzed each of our 26 observations individually, fitting the above-described model to the $0.4-5.0 \mathrm{keV}$ MOS1 and MOS2 spectra simultaneously.

\subsection{Systematic Errors}

Fixing the spectra of the foreground and extragalactic components may introduce systematic errors to our fitting (i.e., the true spectra of these components may differ from our assumed spectra, which may in turn affect the measured halo parameters). Here, we estimate the sizes of these systematic errors.

In the case of the foreground model, we fixed the spectrum because otherwise there would be a degeneracy between the foreground and background intensities. This degeneracy can be overcome by shadowing observations (Smith et al. 2007; Galeazzi et al. 2007; Henley \& Shelton 2008; Gupta et al. 2009; Lei et al. 2009), but such an analysis is not possible here. Here, we fixed the foreground normalization by extrapolating the foreground spectrum from the R12 band into the XMMNewton band. However, as the relative contributions of LB and SWCX emission are likely to differ in these bands, and these two emission mechanisms have different spectra, such an extrapolation may lead to an incorrect estimate of the foreground normalization. We estimated the size of this systematic effect by reanalyzing each sightline, using the median foreground R12 intensity (600 counts $\mathrm{s}^{-1} \operatorname{arcmin}^{-2}$ ) to fix the normalization

\footnotetext{
8 http://heasarc.gsfc.nasa.gov/docs/xanadu/xspec/
}

for every sightline. We used the median differences between our original results and these new results as our estimate of the systematic errors due to the foreground normalization being fixed (we adopted the same systematic errors for each sightline). The estimated systematic errors are $\pm 0.08 \times 10^{6} \mathrm{~K}$ for the halo temperature and \pm 0.04 dex for the halo EM.

We fixed the spectrum of the extragalactic background component because otherwise there would be a degeneracy between this component and the power-law component used to model the soft protons. However, the normalization of this component may vary from field to field, due to statistical fluctuations in the number of unresolved sources that comprise the extragalactic background. We estimated the size of the field-to-field variation in the extragalactic background using the $0.5-2.0 \mathrm{keV}$ source flux distribution from Moretti et al. (2003). Given that we removed sources with fluxes greater than $5 \times 10^{-14} \mathrm{erg} \mathrm{cm}^{-2} \mathrm{~s}^{-1}$, we estimate that the $0.5-2.0 \mathrm{keV}$ extragalactic surface brightness varies by roughly $\pm 10 \%$ from field to field, assuming a typical field of view of $480 \mathrm{arcmin}^{2}$. We therefore repeated our analysis for each sightline with assumed extragalactic normalizations of 9.5 and 11.6 photons $\mathrm{cm}^{-2} \mathrm{~s}^{-1} \mathrm{sr}^{-1} \mathrm{keV}^{-1}$. We used the differences between the original results and these new results to estimate the systematic errors for each sightline due to our fixing the normalization of the extragalactic background. The systematic errors on the halo temperature and EM are typically less than $\pm 0.2 \times 10^{6} \mathrm{~K}$ and \pm 0.2 dex, respectively.

An additional possible source of systematic error is the expected steepening of the extragalactic background below $\sim 2 \mathrm{keV}$. In the $3-10 \mathrm{keV}$ energy range, the extragalactic photon index $\Gamma \approx 1.4$ (Marshall et al. 1980). This photon index also provides good fits to SXRB spectra at lower energies (e.g., Chen et al. 1997, the source of our assumed extragalactic background model). However, the summed spectrum of the individual sources that comprise the extragalactic background is steeper below $\sim 2 \mathrm{keV}$ ( $\Gamma=1.96$; Hasinger et al. 1993). Failing to take this steepening into account would cause us to underestimate the low-energy extragalactic surface brightness and thus overestimate the halo surface brightness. We estimated the size of this systematic effect by using the double broken power-law model for the extragalactic background from Smith et al. (2007). Both broken power laws have $\Gamma_{2}=1.4$ above the break energy of $1.2 \mathrm{keV}$. The first component has $\Gamma_{1}=1.54$ below $1.2 \mathrm{keV}$, and a normalization of 5.70 photons $\mathrm{cm}^{-2}$ $\mathrm{s}^{-1} \mathrm{sr}^{-1} \mathrm{keV}^{-1}$. The second component has $\Gamma_{1}=1.96$ and a nominal normalization of 4.90 photons $\mathrm{cm}^{-2} \mathrm{~s}^{-1} \mathrm{sr}^{-1} \mathrm{keV}^{-1}$. Note that the second normalization was a free parameter in Smith et al.'s (2007) analysis, and they obtained a value roughly $50 \%$ lower. However, because of the aforementioned degeneracy between the extragalactic background and the softproton component, we fix the normalization at its nominal value. Note also that the spectrum of the extragalactic background depends on the flux level to which sources are removed; it is not clear to what source removal threshold the Smith et al. (2007) model is applicable. Nevertheless, we proceeded by repeating our analysis for each sightline, using this new extragalactic background model. As with the normalization of the extragalactic background, we used the differences between the original results and these new results to estimate the systematic errors for each sightline. The systematic errors on the halo temperature are typically less than $\pm 0.1 \times 10^{6} \mathrm{~K}$. As expected, this new extragalactic model generally yields lower halo EMs, although the difference is typically less than 0.2 dex. 
Table 2

Spectral Fit Results

\begin{tabular}{|c|c|c|c|c|c|c|c|}
\hline No. & $\begin{array}{l}\text { Obs. ID } \\
\text { (1) }\end{array}$ & $\begin{array}{c}\text { Foreground R12 Rate } \\
\text { (ROSAT Units) } \\
\text { (2) }\end{array}$ & $\begin{array}{c}N_{\mathrm{H}} \\
\left(10^{20} \mathrm{~cm}^{-2}\right) \\
(3)\end{array}$ & $\begin{array}{c}\text { Halo } T \\
\left(10^{6} \mathrm{~K}\right) \\
(4)\end{array}$ & $\begin{array}{c}\text { Halo EM } \\
\left(10^{-3} \mathrm{~cm}^{-6} \mathrm{pc}\right) \\
(5)\end{array}$ & $\begin{array}{c}\chi^{2} / \text { dof } \\
(6)\end{array}$ & $\begin{array}{c}S_{0.4-2.0} \\
\left(10^{-12} \mathrm{erg} \mathrm{cm}^{-2} \mathrm{~s}^{-1} \mathrm{deg}^{-2}\right) \\
(7)\end{array}$ \\
\hline 1 & 0304070501 & 743 & 0.790 & $2.31_{-0.24-0.24}^{+0.08+0.16}$ & $1.63_{-0.25}^{+0.20}+0.29$ & $405.97 / 362$ & 1.66 \\
\hline 2 & 0305290201 & 293 & 5.83 & $2.19_{-0.08}^{+0.07}{ }_{-0.16}^{+0.21}$ & $3.96_{-0.45}^{+0.27}+0.78$ & $475.90 / 440$ & 3.73 \\
\hline 3 & 0401210601 & 593 & 3.45 & $2.25_{-0.23}^{+0.11}{ }_{-0.21}^{+0.16}$ & $1.80_{-0.34}^{+0.27}{ }_{-0.52}^{+0.45}$ & $452.03 / 395$ & 1.75 \\
\hline 4 & 0404220101 & 606 & 1.72 & $1.95_{-0.19}^{+0.20}+0.72$ & $1.78_{-0.52}^{+0.18}-0.32$ & $469.00 / 422$ & 1.40 \\
\hline 5 & 0400560301 & 643 & 1.60 & $2.10_{-0.06}^{+0.05} \pm 0.16$ & $3.05_{-0.19}^{+0.17}{ }_{-0.64}^{0.56}$ & $693.09 / 594$ & 2.71 \\
\hline 6 & 0400570201 & 586 & 0.642 & $2.08_{-0.13}^{+0.09}{ }_{-0.24}^{+0.17}$ & $1.79_{-0.19}^{+0.23}{ }_{-0.53}^{+0.40}$ & $504.83 / 529$ & 1.57 \\
\hline 7 & 0406630201 & 577 & 1.00 & $11.18_{-0.50-1.41}^{+1.04}$ & $1.64_{-0.49}^{+0.16}{ }_{-0.56}^{+0.29}$ & $285.43 / 286$ & 1.89 \\
\hline 8 & 0303260201 & 555 & 0.640 & $1.83_{-0.06}^{+0.02}{ }_{-0.17}^{0.16}$ & $4.49_{-0.33}^{+0.14} \pm 1.01$ & $607.54 / 593$ & 3.05 \\
\hline 9 & 0306060201 & 687 & 1.26 & $1.46_{-0.04}^{+0.03}{ }_{-0.16}^{+0.49}$ & $\begin{array}{l}5.54_{-0.52}^{+2.27}+0.98 \\
-3.35\end{array}$ & $587.85 / 594$ & 2.13 \\
\hline 10 & 0306060301 & 687 & 1.26 & $2.06_{-0.13}^{+0.08}{ }_{-0.21}^{0.16}$ & $3.01_{-0.49}^{+0.16}{ }_{-0.57}^{0.54}$ & $405.23 / 412$ & 2.60 \\
\hline 11 & 0303720601 & 641 & 1.21 & $2.11_{-0.09}^{+0.12}+0.32$ & $2.16_{-0.25}^{+0.26}+1.09$ & $498.38 / 445$ & 1.93 \\
\hline 12 & 0303720201 & 641 & 1.21 & $2.08_{-0.06}^{+0.08}{ }_{-0.16}^{+0.17}$ & $3.07_{-0.36}^{+0.22}{ }_{-0.53}^{+0.88}$ & $494.35 / 494$ & 2.68 \\
\hline 13 & 0200960101 & 615 & 1.94 & $2.20_{-0.08}^{+0.05} \pm 0.17$ & $2.12_{-0.19}^{+0.11}{ }_{-0.45}^{+0.39}$ & $606.12 / 594$ & 2.01 \\
\hline 14 & 0301340101 & 575 & 3.31 & $1.99_{-0.11}^{+0.06} \pm 0.16$ & $3.35_{-0.42}^{+0.29}{ }_{-0.64}^{+0.74}$ & $349.15 / 403$ & 2.73 \\
\hline 15 & 0306370601 & 742 & 0.656 & $1.72_{-0.10}^{+0.12}{ }_{-0.18}^{+0.16}$ & $3.55_{-0.68}^{+0.35}{ }_{-0.58}^{0.81}$ & $353.40 / 340$ & 2.11 \\
\hline 16 & 0402780701 & 471 & 4.54 & $2.03_{-0.48}^{+0.37}+9.89$ & $0.69_{-0.36}^{+0.37}{ }_{-0.45}^{+0.56}$ & $400.90 / 451$ & 0.58 \\
\hline 17 & 0304203401 & 763 & 1.19 & $1.96_{-0.10}^{+0.09}+0.17$ & $4.97_{-0.54}^{+0.47}{ }_{-1.16}^{0.88}$ & $271.14 / 239$ & 3.91 \\
\hline 18 & 0406610101 & 806 & 1.65 & $3.14_{-0.23}^{+0.42}{ }_{-0.22}^{+0.61}$ & $2.45_{-0.69}^{+0.23}{ }_{-0.92}^{0.44}$ & $367.42 / 368$ & 3.25 \\
\hline 19 & 0400830301 & 527 & 1.74 & $1.91_{-0.06}^{+0.10}-0.22$ & $2.39_{-0.17-0.78}^{+0.28}+0.79$ & $630.86 / 594$ & 1.80 \\
\hline 20 & 0301651701 & 542 & 1.73 & $1.83 \pm 0.05 \pm 0.16$ & $6.79_{-0.46-1.39}^{+0.37}$ & $386.43 / 376$ & 4.65 \\
\hline 21 & 0300630301 & 698 & 2.13 & $1.99_{-0.36-0.19}^{+0.26}$ & $1.03_{-0.32}^{+0.43}{ }_{-0.32}^{+0.58}$ & $380.59 / 413$ & 0.84 \\
\hline 22 & 0312190601 & 427 & 2.29 & $2.28_{-0.21}^{+0.13} \pm 0.19$ & $1.85_{-0.39}^{+0.25}{ }_{-0.72}^{+0.73}$ & $322.29 / 326$ & 1.84 \\
\hline 23 & 0301330401 & 417 & 2.73 & $2.19_{-0.16}^{+0.11}{ }_{-0.16}^{+0.41}$ & $1.63_{-0.24}^{+0.25}{ }_{-0.84}^{0.36}$ & $457.39 / 496$ & 1.54 \\
\hline 24 & 0312190701 & 573 & 1.75 & $2.04_{-0.10}^{+0.07}{ }_{-0.19}^{+0.16}$ & $3.78_{-0.33}^{+0.31}{ }_{-0.85}^{+1.04}$ & $375.97 / 363$ & 3.21 \\
\hline 25 & 0302500101 & 597 & 3.01 & $2.35_{-0.06}^{+0.03}+0.19$ & $5.27_{-0.36}^{+0.13}+0.94$ & $446.89 / 476$ & 5.48 \\
\hline 26 & 0307001401 & 666 & 2.63 & $3.31_{-1.09-0.47}^{+0.90}$ & $0.37_{-0.23}^{+0.19}{ }_{-0.29}^{+0.07}$ & $380.72 / 335$ & 0.51 \\
\hline
\end{tabular}

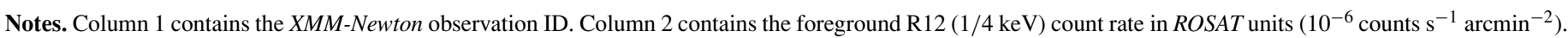
This count rate was derived from the Snowden et al. (2000) catalog of SXRB shadows, and was used to fix the normalization of the $1.2 \times 10^{6} \mathrm{~K}$ foreground component. Column 3 contains the H I column density (Kalberla et al. 2005) that was used to attenuate the halo and extragalactic components. Columns 4 and 5 contain the best-fit halo parameters $\left(\mathrm{EM}=\int n_{\mathrm{e}}^{2} d l\right)$. In each case, the first error indicates the $1 \sigma$ statistical error, and the second error indicates the estimated systematic error due to our assumed foreground and extragalactic spectra (see Section 3.2 for details). Column 6 contains $\chi^{2}$ and the number of degrees of freedom. Column 7 contains the intrinsic $0.4-2.0 \mathrm{keV}$ surface brightness of the halo component.

To calculate the systematic errors on the halo parameters for each sightline, we added the three systematic errors discussed above in quadrature. We report these combined systematic errors alongside the statistical errors in the following section.

\subsection{Spectral Fit Results}

The results of our spectral analysis are presented in Table 2. In particular, Columns 4 and 5 give the best-fitting halo temperature and EM for each observation, and Column 7 gives the intrinsic 0.4-2.0 keV surface brightness of the halo component. For the temperature and the EM, we present both the $1 \sigma$ statistical errors and the estimated systematic errors discussed in the previous section.

The best-fit halo parameters are plotted against Galactic latitude in Figure 2. The error bars show the statistical and systematic errors added in quadrature. The observations with large temperature error bars at $|b| \approx 33^{\circ}$ and $35^{\circ}$ are 0402780701 and 0307001401 (numbers 16 and 26, respectively, in Tables 1 and 2). Note that the halo is faint in these directions (indeed, these observations yield the two lowest $0.4-2.0 \mathrm{keV}$ surface brightnesses), and that the exposure times are not unusually long. It is therefore unsurprising that the halo temperatures are poorly constrained for these observations. The other temperatures are generally well constrained; they are typically $\sim(1.8-2.4) \times 10^{6} \mathrm{~K}$, and are fairly constant across the sky. The halo EMs mostly lie in the range $\sim 0.0005-0.006 \mathrm{~cm}^{-6} \mathrm{pc}$, although there is significant variation within that range. Correspondingly, there is also significant variation in the intrinsic $\mathrm{X}$-ray surface brightness, within the range $\sim(0.5-5) \times 10^{-12} \mathrm{erg} \mathrm{cm}^{-2} \mathrm{~s}^{-1} \mathrm{deg}^{-2}$.

One observation, 0406630201 (number 7 in Table 2), gives a significantly higher halo temperature than the other observations $\left(11.2 \times 10^{6}\right.$ versus $\left.\sim 2 \times 10^{6} \mathrm{~K}\right)$. The MOS2 spectrum and best-fit model for this observation are shown in Figure 3. For comparison, Figure 3 also shows the spectrum from a nearby observation (0303260201; number 8 in Table 2) that yields a more typical halo temperature $\left(1.8 \times 10^{6} \mathrm{~K}\right)$. The halo component for obs. 0406630201 is faint, and the temperature may be less well constrained than the formally calculated error bar implies. We tried repeating our analysis of this observation with the halo temperature fixed at $2 \times 10^{6} \mathrm{~K}$ (i.e., similar to the temperatures found from most of the other observations). The best-fit EM for this new halo component was essentially 

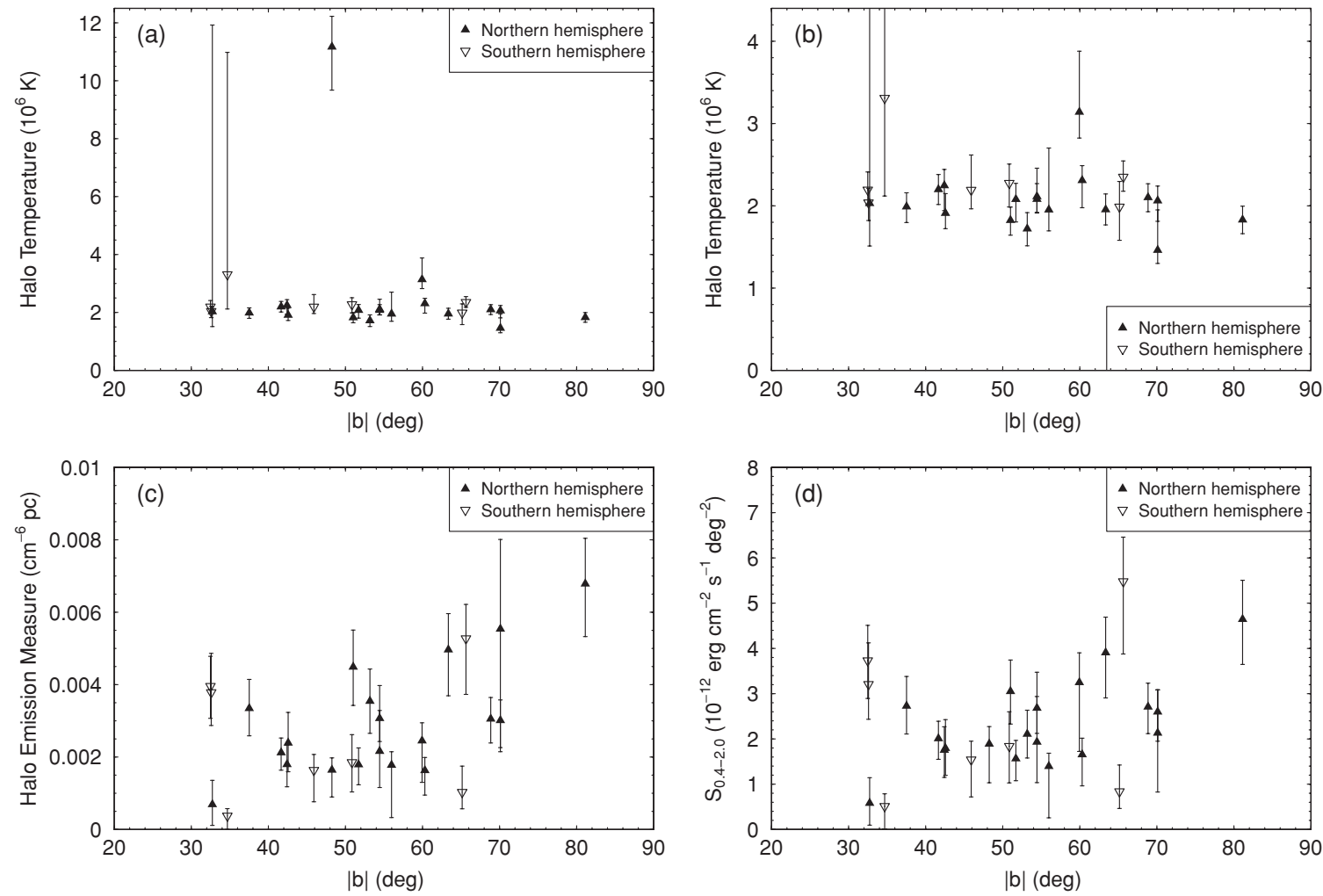

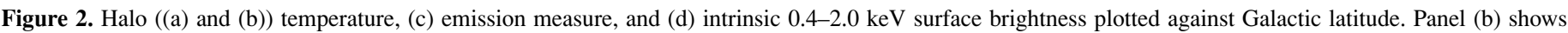

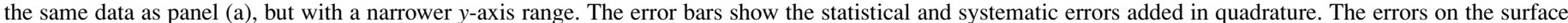
brightness are derived from the errors on the emission measure.
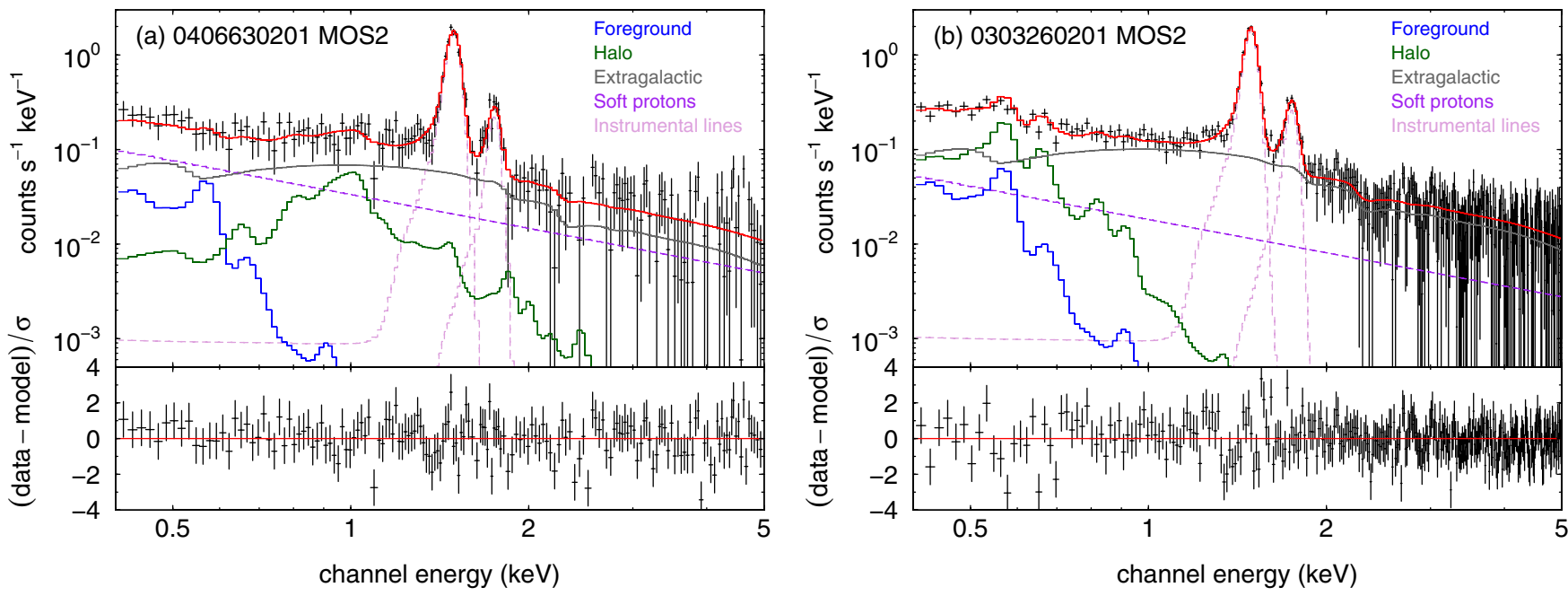

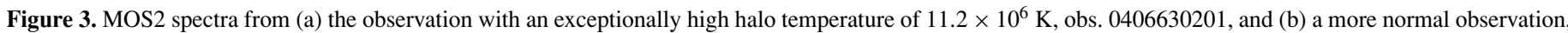

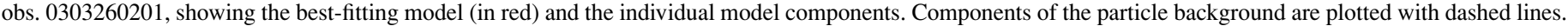

zero ( $3 \sigma$ upper limit: $0.0022 \mathrm{~cm}^{-6} \mathrm{pc}$ ). This new model yielded an acceptable fit: $\chi^{2}=309.59$ for 287 degrees of freedom. Therefore, although the formal best-fitting temperature is $11.2 \times 10^{6} \mathrm{~K}$, this spectrum is also consistent with a $\sim 2 \times 10^{6} \mathrm{~K}$ halo with a small EM $\left(\lesssim 0.002 \mathrm{~cm}^{-6} \mathrm{pc}\right)$. The upper limit on the EM is not unusually small compared with some of the other observations.

Although there is this one anomalous temperature among our 26 XMM-Newton observations, it should not affect our subsequent analysis. In Section 4, we will compare our observations of the halo with the predictions of various physical models. Rather than looking at individual sightlines, we look at the whole pop- ulation of observational results, and compare histograms of observational properties with corresponding histograms derived from the models. Therefore, as long as the majority of our observations yield reasonably accurate halo properties, a single outlying anomalous result should not significantly affect the comparison of the observations with the models.

\section{COMPARING THE OBSERVED HALO X-RAY SPECTRA WITH HYDRODYNAMICAL MODELS}

In this section, we compare the X-ray spectral properties of the halo inferred from our XMM-Newton observations with those predicted by various hydrodynamical models. In particular, in 


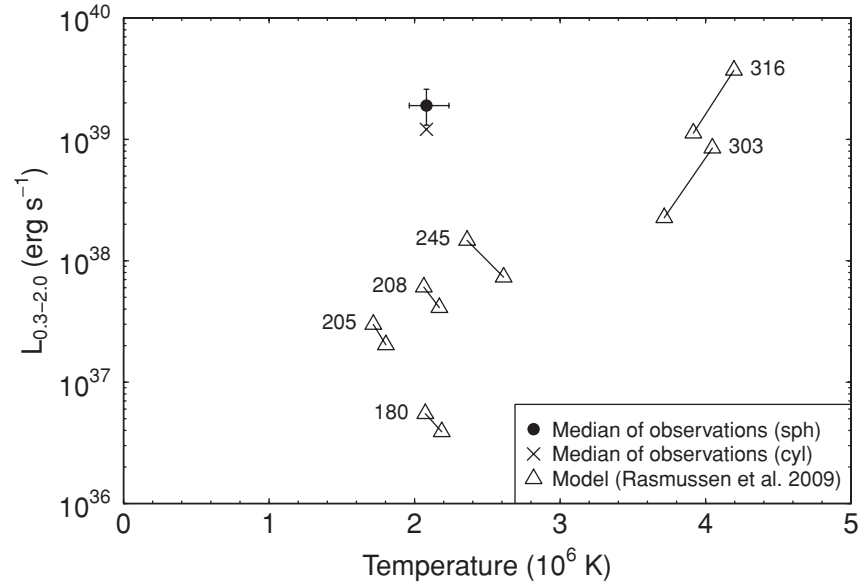

Figure 4. Halo temperatures and $0.3-2.0 \mathrm{keV}$ luminosities derived from our XMM-Newton observations and from disk galaxy formation simulations (Rasmussen et al. 2009). The triangles show the emission-weighted mean temperatures and halo luminosities predicted by the model. These data points are labeled with the model galaxies' circular velocities, $v_{\mathrm{c}}$, in $\mathrm{km} \mathrm{s}^{-1}$. For each model galaxy, the lower data point shows the values extracted from within a spherical region of radius $40 \mathrm{kpc}$, and the upper data point shows the values extracted from within a $(100 \mathrm{kpc})^{3}$ box. In both cases, a cylindrical region around the galactic disk was excluded. The solid circle and the diagonal cross show the median observed values. The solid circle denotes the median halo luminosity derived from the observations assuming a spherical emission geometry (Equation (3)); the error bars indicate the lower and upper quartiles. The diagonal cross denotes the median halo luminosity derived assuming a cylindrical emission geometry (Equation (4)).

Section 4.1 we examine a disk galaxy formation model, in which extragalactic material is heated as it falls into the Galaxy's potential well (Toft et al. 2002; Rasmussen et al. 2009). In Section 4.2, we examine a model in which the hot halo gas is heated in situ by isolated extraplanar SNe (Shelton 2006). In Section 4.3, we examine a model in which the ISM is heated and stirred by multiple SNe (Joung \& Mac Low 2006). Unlike the previous model, the SNRs are not assumed to evolve in isolation, and the model includes the movement of hot gas from the disk into the halo.

\subsection{Disk Galaxy Formation Model}

Cosmological smoothed particle hydrodynamics (SPH) simulations of disk galaxy formation predict that such galaxies should be surrounded by extended hot halos ( $r \sim$ tens of kpc, $T \sim$ few $\times 10^{6} \mathrm{~K}$; Toft et al. 2002; Rasmussen et al. 2009). These halos contain a significant fraction of the galactic baryonic mass (Sommer-Larsen 2006). In this subsection, we compare the predictions of such models with our XMM-Newton observations.

J. Rasmussen (2009, private communication) has kindly provided us with $0.3-2.0 \mathrm{keV}$ luminosities and emissionweighted mean temperatures derived from the SPH galaxy formation simulations described in Rasmussen et al. (2009). Rasmussen et al. (2009) point out that the X-ray emission from the hot gas particles can be artificially boosted by nearby cold, dense gas particles (within an SPH smoothing length, $h_{\mathrm{SPH}}$ ). As $h_{\mathrm{SPH}} \approx 1.5 \mathrm{kpc}$ in these simulations, the emission from the disk can be particularly adversely affected. Therefore, a cylindrical region around each galactic disk within $|z|=2 \mathrm{kpc}$ and $r=15 \mathrm{kpc}$ was excluded from the calculation of the X-ray properties. For each galaxy, two sets of values were extracted: one extracted from within a spherical region of radius $40 \mathrm{kpc}$ (these are the values shown in Figures 5 and 6 in Rasmussen et al. 2009) and the other extracted from within a $(100 \mathrm{kpc})^{3}$ box. The X-ray luminosities and temperatures for each model galaxy are shown by the triangles in Figure 4.

In order to compare the model predictions with our observations, we must first derive a luminosity for the Galactic halo from our observations. To do this, we must assume some geometry for the halo emission. In the following, we consider both spherical and cylindrical geometries. Of the two, a spherical geometry is probably the more appropriate for comparison with the extended hot halo predicted by the model, especially as a cylindrical region around each model galactic disk was excluded before the X-ray properties were extracted.

For the spherical halo geometry, we assume that the halo emission comes from a uniform sphere of radius $R_{\mathrm{sph}}$. If the intrinsic surface brightness, $S_{\mathrm{X}}$, along some sightline is typical for the whole galaxy, the luminosity per unit volume of the halo is $4 \pi S_{\mathrm{X}} / \lambda$, where $\lambda$ is the path length through the spherical halo. This path length is a function of the viewing direction:

$$
\lambda=R_{\odot} \cos l \cos b+\sqrt{R_{\mathrm{sph}}^{2}-\left(1-\cos ^{2} l \cos ^{2} b\right) R_{\odot}^{2}},
$$

where $R_{\odot}=8.5 \mathrm{kpc}$ is the radius of the solar circle. The X-ray luminosity is then given by

$$
L_{\mathrm{X}}=\frac{16 \pi^{2}}{3} \frac{R_{\mathrm{sph}}^{3} S_{\mathrm{X}}}{\lambda} .
$$

For the cylindrical halo geometry, if the intrinsic surface brightness along some sightline is typical for the whole galaxy, the luminosity per unit area of the halo integrated over the vertical direction is $2 \times 4 \pi \sin (|b|) S_{\mathrm{X}}$. The initial factor of two takes into account the halo above and below the disk. If we then assume that the halo emission originates within a cylindrical region of radius $R_{\text {cyl }}$, the total X-ray luminosity of the Galactic halo is

$$
L_{\mathrm{X}}=8 \pi^{2} \sin (|b|) R_{\mathrm{cyl}}^{2} S_{\mathrm{X}} .
$$

We have calculated intrinsic $0.3-2.0 \mathrm{keV}$ surface brightnesses for the halo from our best-fitting spectral models in Table 2. We then converted each surface brightness to a $0.3-2.0 \mathrm{keV}$ luminosity, using Equations (3) and (4). In both cases, we assumed emission radii $\left(R_{\mathrm{sph}}\right.$ or $\left.R_{\mathrm{cyl}}\right)$ of $15 \mathrm{kpc}$. There is a large amount of scatter in the derived luminosities, spanning about an order of magnitude. The medians of these values are $1.9 \times 10^{39} \mathrm{erg} \mathrm{s}^{-1}$ (spherical geometry) and $1.2 \times 10^{39} \mathrm{erg} \mathrm{s}^{-1}$ (cylindrical geometry); these are our best estimates of the 0.3-2.0 keV luminosity of the Milky Way halo. However, it should be noted that our sightlines are all in directions away from the Galactic Center, and so if the halo emissivity increases toward the Galactic Center, the above values will underestimate the Galactic luminosity. In addition, the luminosity inferred assuming a spherical geometry will be an underestimate if the halo is more extended than our assumed $R_{\mathrm{sph}}=15 \mathrm{kpc}$ (note from Figure 4 that the model predicts that a significant fraction of the halo emission originates from $r>40 \mathrm{kpc}$ ). For comparison, analysis of ROSAT All-Sky Survey data has yielded halo Xray luminosities of $7 \times 10^{39} \mathrm{erg} \mathrm{s}^{-1}$ in the $0.1-2.0 \mathrm{keV}$ band (assuming that the quoted value applies to the whole ROSAT band; Pietz et al. 1998) and $3 \times 10^{39} \mathrm{erg} \mathrm{s}^{-1}$ in the $0.5-2.0 \mathrm{keV}$ band (Wang 1998). Assuming a halo temperature of $2 \times 10^{6} \mathrm{~K}$, these values correspond to $0.3-2.0 \mathrm{keV}$ luminosities of $4 \times 10^{39}$ and $5 \times 10^{39} \mathrm{erg} \mathrm{s}^{-1}$, respectively.

Figure 4 compares the median observed halo temperature and the above-mentioned median halo luminosities with the predictions of the disk galaxy formation simulations. For model 
galaxies similar in size to the Milky Way $\left(v_{\mathrm{c}} \approx 220 \mathrm{~km} \mathrm{~s}^{-1}\right)$, the predicted temperatures are in good agreement with the observations. However, the Rasmussen et al. (2009) model underpredicts the observed halo luminosity by at least an order of magnitude. We will discuss these results in Section 5.2.1.

\subsection{Extraplanar Supernova Explosions}

Here, we consider a model in which the hot halo gas is heated locally by $\mathrm{SNe}$ above the Galactic disk. In this scenario, the observed hot gas is within isolated SNRs at a variety of heights above the disk and of a variety of ages. Superbubbles blown by clustered $\mathrm{SNe}$ and hot gas that has risen from the disk are excluded in this model. This model was developed by Shelton (2006), who found that it could explain a significant fraction of the high-latitude $1 / 4 \mathrm{keV}$ halo emission (excluding anomalously bright features such as the North Polar Spur). Here, we compare the model to the higher-energy emission observed with $X M M$ Newton.

\subsubsection{Model Description}

The model spectra discussed here were generated from onedimensional Lagrangian hydrodynamical simulations of SNRs evolving at a variety of heights above the Galactic disk, and hence in a variety of ambient densities. The simulations are described fully in Shelton (1998, 1999, 2006), and include radiative cooling, thermal conduction, and an effective ambient magnetic field, $B_{\text {eff }}$, which exerts a non-thermal pressure, in addition to the ambient gas pressure. The ionization evolution in the shocked gas is modeled self-consistently.

We will first concentrate on the models from Shelton (2006) with $\mathrm{SN}$ explosion energy $E_{0}=0.5 \times 10^{51}$ erg and $B_{\text {eff }}=$ $2.5 \mu \mathrm{G}$ (corresponding to a non-thermal pressure $P_{\mathrm{nt}}=$ $1800 \mathrm{~cm}^{-3} \mathrm{~K}$ ), and then discuss varying these parameters. We consider the models evolving in ambient densities $n_{0}=0.2$, $0.1,0.05,0.02,0.01$, and $0.005 \mathrm{~cm}^{-3}$, corresponding to heights $z=190,310,480,850,1300$, and $1800 \mathrm{pc}$, using the interstellar density model from Ferrière (1998). Note that we are ignoring the model from Shelton (2006) at $z=76 \mathrm{pc}$, as we do not consider this to be in the halo. Density and temperature profiles from the model with $n_{0}=0.01 \mathrm{~cm}^{-3}$ are shown in Figure 5. The SN explosion blows a hot rarefied bubble in the ambient medium; this hot bubble is the source of the X-rays. The model shown in Figure 5 corresponds to model A in Shelton (1999); see that paper for more details.

For each epoch of each SNR model, we calculated spectra for a range of impact parameters through the model remnant, using the Raymond \& Smith (1977) spectral code (updated by J. C. Raymond \& B. W. Smith 1993, private communication with R. J. Edgar). The spacing between the impact parameters, $\Delta b$, was adjusted according to the size of the remnant. The spectral calculation takes into account the possible non-equilibrium ionization calculated during the hydrodynamical simulation.

We wish to compare the distributions of observed temperatures and EMs with those predicted by the model. In order to do this, we need to calculate the probability of a sightline passing through a remnant at a given height, of a given age, and at a given impact parameter. For example, a sightline is more likely to pass through a remnant at a larger impact parameter than a small impact parameter, and more likely to pass through a remnant closer to the disk, where the $\mathrm{SN}$ rate is larger. If $R\left(z_{1}, z_{2}\right)$ is the rate per unit area of SNe at heights between $z_{1}$ and $z_{2}$, then the probability $P\left(z_{1}, z_{2}, t, \Delta t, b, \Delta b\right)$ of intercepting a remnant

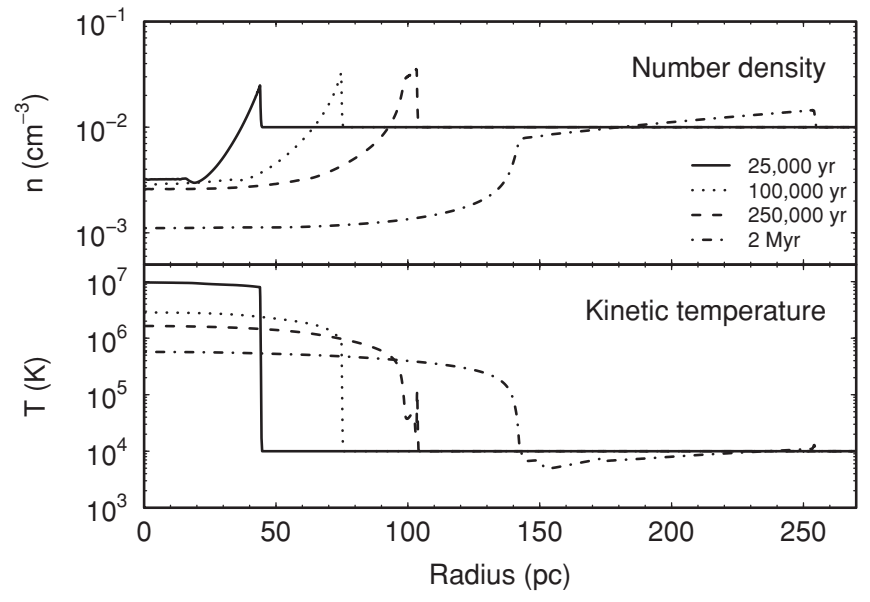

Figure 5. Density and temperature profiles for an SNR model with $E_{0}=$ $0.5 \times 10^{51} \mathrm{erg}, B_{\text {eff }}=2.5 \mu \mathrm{G}$ evolving in an ambient medium with $n_{0}=0.01 \mathrm{~cm}^{-3}$. Note that the cool shell has formed by $250,000 \mathrm{yr}$.

between these heights with an age between $t$ and $t+\Delta t$ at an impact parameter between $b$ and $b+\Delta b$ is

$$
P\left(z_{1}, z_{2}, t, \Delta t, b, \Delta b\right)=2 \pi b R\left(z_{1}, z_{2}\right) \Delta b \Delta t .
$$

In order to calculate the above probabilities, we followed Shelton (2006) and divided the halo into six plane-parallel slabs. We assumed that all the SNRs within a given slab experience a single ambient density, and so can be represented by one of our six SNR models. We calculated $R\left(z_{1}, z_{2}\right)$ for each slab by integrating the volumetric $\mathrm{SN}$ rate at the solar circle, $r(z)$, from Ferrière (1998):

$$
\begin{aligned}
r(z) & =r_{\mathrm{Ia}}(z)+r_{\mathrm{II}}(z) \\
& =\left(4.0 e^{-|z| / 325 \mathrm{pc}}+14 e^{-|z| / 266 \mathrm{pc}}\right) \mathrm{kpc}^{-3} \mathrm{Myr}^{-1},
\end{aligned}
$$

where $r_{\mathrm{Ia}}(z)$ and $r_{\mathrm{II}}(z)$ are the rates of Type Ia SNe and isolated core-collapse $\mathrm{SNe}$, respectively. The slab boundaries were placed at the midpoints between the nominal heights of the model remnants, except for the lower boundary of the lowest slab, which was placed at $130 \mathrm{pc}$ (the scale height of the Galactic H I layer), and the upper boundary of the highest slab, which was placed at infinity. The boundaries, ambient densities, and $\mathrm{SN}$ rates for the six slabs are shown in Table 3. We calculated $\Delta t$ from the timestamps of the output files from the hydrodynamical simulations. As noted above, $\Delta b$ is the spacing between the impact parameters for which we calculated spectra from a given remnant.

We used a Monte Carlo method to construct model spectra for 2000 sightlines, using the above-calculated probabilities. In our Monte Carlo simulation, $64 \%$ of the model sightlines intercepted no remnants, and $9 \%$ of the sightlines intercepted more than one remnant. For model sightlines intercepting more than one remnant, we summed the spectra of the individual remnants.

\subsubsection{Characterizing the SNR Spectra with $1 T$ Models}

In our analysis of the XMM-Newton observations, we modeled the halo X-ray emission with a single-temperature CIE plasma model, whereas the true halo emission is likely from plasma at a range of temperatures and in a range of ionization states. Similarly, the emission predicted by this extraplanar SNR model is from plasma at a range of temperatures and in a range of 
Table 3

Densities and Supernova Rates in the Halo as a Function of Height

\begin{tabular}{lcccc}
\hline \hline Slab & $\begin{array}{c}\text { Height Range } \\
(\mathrm{pc})\end{array}$ & $\begin{array}{c}\text { Ambient Density, } n_{0} \\
\left(\mathrm{~cm}^{-3}\right)\end{array}$ & $\begin{array}{c}\text { Nominal SNR Height } \\
(\mathrm{pc})\end{array}$ & $\begin{array}{c}\text { SN Rate, } R \\
\left(\mathrm{kpc}^{-2} \mathrm{Myr}^{-1}\right)\end{array}$ \\
\hline 1 & $130-250$ & 0.2 & 190 & 1.1 \\
2 & $250-395$ & 0.1 & 310 & 0.83 \\
3 & $395-665$ & 0.05 & 480 & 0.76 \\
4 & $665-1075$ & 0.02 & 850 & 0.36 \\
5 & $1075-1550$ & 0.01 & 1300 & 0.091 \\
6 & $1550-\infty$ & 0.005 & 1800 & 0.022 \\
\hline
\end{tabular}

ionization states. Therefore, in order to compare the predictions of this model with our observational results, we first characterize the model SNR spectra calculated above with $1 T$ CIE plasma models, by simulating XMM-Newton observations of the SXRB.

Our procedure for characterizing the spectrum for each model sightline is as follows. We first multiplied the spectrum by a renormalization factor, $k_{\mathrm{rn}}$, in order to give a $0.4-2.0 \mathrm{keV}$ surface brightness of $2.06 \times 10^{-12} \mathrm{erg} \mathrm{cm}^{-2} \mathrm{~s}^{-1} \mathrm{deg}^{-2}$. This value is the median intrinsic halo surface brightness inferred from the best-fit models in Table 2 . We then subjected the model to an absorbing column $N_{\mathrm{H}}=1.7 \times 10^{20} \mathrm{~cm}^{-2}$; again, this is the median value used in our XMM-Newton analysis. To the absorbed SNR spectrum, we added components representing the foreground emission, the extragalactic background, the soft protons, and the instrumental lines. The parameters for the foreground emission and the extragalactic background were taken from our observational analysis (see Section 3.1). The normalization of the foreground component was chosen to give an R12 count rate of 600 counts s${ }^{-1}$ arcmin $^{-2}$ (the median value from Table 2). The parameters for the soft-proton model and the instrumental lines were taken from the best-fit model for obs. 0301330401; this observation was chosen because it has close to the median level of soft-proton contamination, as judged by the ratio of the observed $2-5 \mathrm{keV}$ flux to that expected from the extragalactic background $\left(F_{\text {total }}^{2-5} / F_{\text {exgal }}^{2-5}\right.$; see Paper I).

We simulated an observation of the model spectrum by folding the above-described multicomponent model through the XMM-Newton response, assuming a typical field of view of $480 \mathrm{arcmin}^{2}$, and adding Poissonian noise to the spectrum, assuming a typical observing time of $15 \mathrm{ks}$. Our simulations also took into account the QPB spectrum. For each model sightline, we simulated a MOS1 and a MOS2 spectrum. We binned the resulting spectra such that there were at least 25 counts per bin. Note that our assumed field of view is not as large as the full $X M M$-Newton field of view, as we removed bright sources from the fields of our observations, and for some observations not all chips were usable.

We fitted the resulting simulated spectra with the same multicomponent model that we used in our observational analysis. In particular, the halo emission was modeled with an absorbed Raymond \& Smith model, with $N_{\mathrm{H}}$ fixed at $1.7 \times 10^{20} \mathrm{~cm}^{-2}$. For each model sightline, we fitted to the simulated MOS1 and MOS2 spectra simultaneously. Figure 6 shows a simulated $X M M$-Newton spectrum from one of our model sightlines, along with the best-fitting model. Also shown is the $1 T$ CIE halo component of the best-fitting model, as well as the input SNR model. The input SNR spectrum is very different from that of a CIE plasma. The SNR plasma is relatively cool (as shown by the differential EM, plotted as the solid line in Figure 7) and overionized, and the spectrum exhibits strong recombination edges. If the SNR plasma were in CIE, instead of recombining, its emission would be four orders of magnitude fainter in the

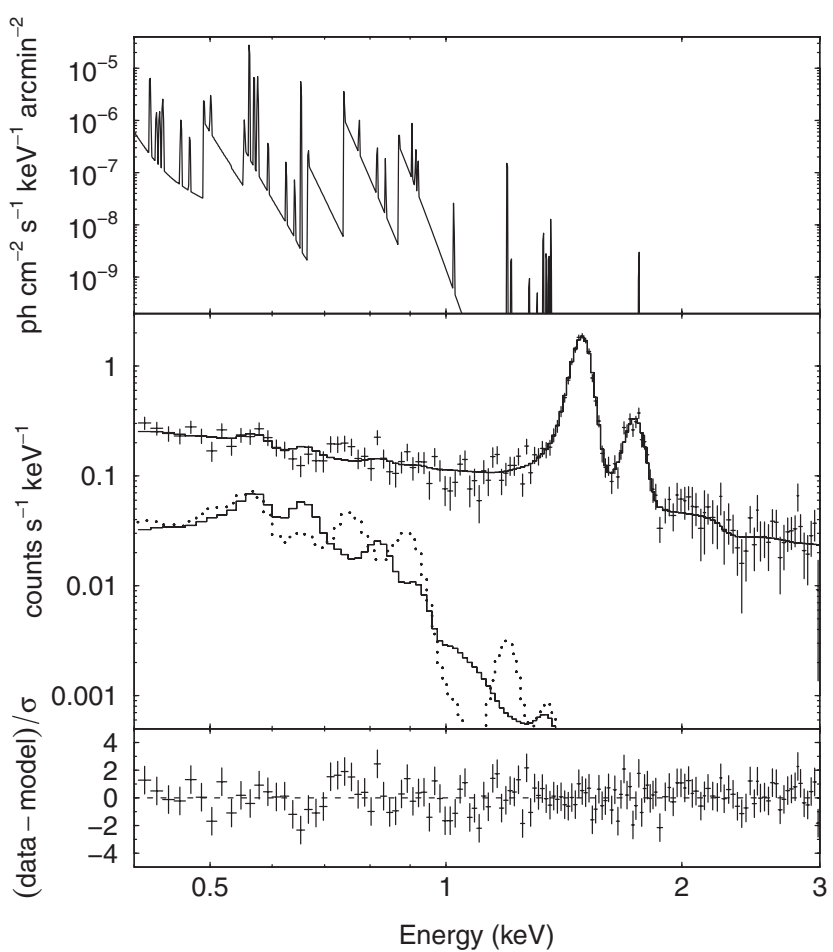

Figure 6. Simulated XMM-Newton MOS1 spectrum of the SXRB, created using the extraplanar SNR model for the halo emission. The top panel shows the input SNR spectrum. The middle panel shows the simulated SXRB spectrum (crosses). The upper solid line shows the best-fit SXRB model, while the lower solid line shows the $1 T$ halo component from that model (the other model components have been omitted for clarity; cf. Figure 3). The dotted line shows the input SNR spectrum folded through the XMM-Newton response. The bottom panel shows the residuals.

XMM-Newton band. However, when we use a $1 T$ CIE plasma to model the halo component in our simulated spectra, the fits are generally good. This is because much of the spectral detail in the input SNR spectrum is lost when it is combined with the other components of the SXRB and folded through the XMM-Newton response. There is some excess emission at $\sim 0.75 \mathrm{keV}$ in the simulated spectrum in Figure 6, possibly due to $\mathrm{O}^{+7} \rightarrow \mathrm{O}^{+6}$ recombinations (the recombination edge is at $0.74 \mathrm{keV}$ ), but it would not be easy to unambiguously identify such a feature in an observed spectrum as recombination emission.

We used the resulting fit parameters to compare with the observations. The temperatures were taken directly from the fits, while the best-fit EMs were first divided by the relevant values of $k_{\mathrm{rn}}$ before comparing with the observations. In what follows, we refer to these values as "X-ray temperatures" and "X-ray EMs," to emphasize that they are derived from simulated $\mathrm{X}$-ray observations, rather than being derived directly from the hydrodynamical data. 


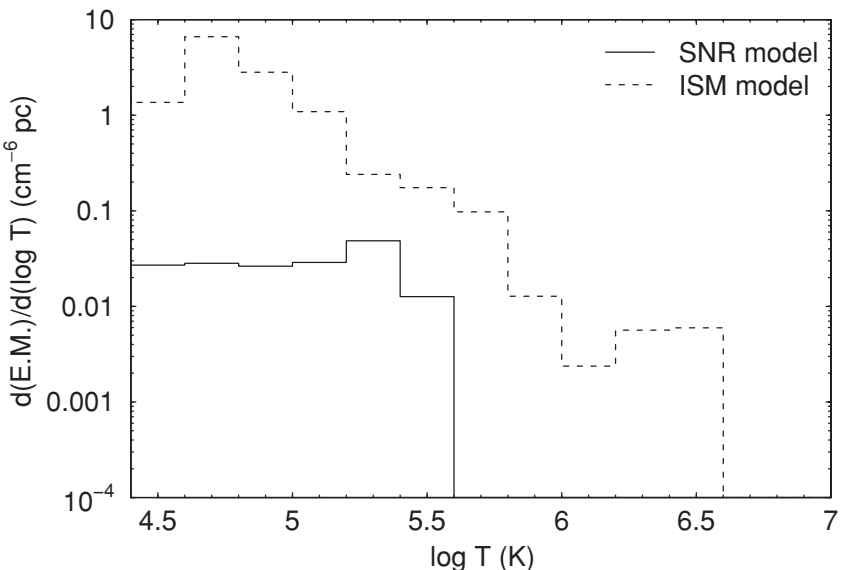

Figure 7. Differential emission measures for the input SNR model plotted in Figure 6 (solid line) and the input SN-driven ISM model plotted in Figure 10 (dashed line).

\subsubsection{Comparing the Extraplanar SNR Model with Observations}

Our model X-ray EMs were derived for sightlines looking straight upward from the disk, i.e., toward $|b|=90^{\circ}$. Our observations, however, are toward a range of Galactic latitudes, and so will sample different amounts of halo material. We therefore multiply our observed EMs by $\sin |b|$ before comparing them with the model predictions. This transformation assumes that the halo is, in a statistical sense at least, uniform in directions parallel to the disk.

Figures 8(a) and (b) show histograms comparing the halo $\mathrm{X}$-ray temperatures and EMs predicted by the extraplanar SNR model with the corresponding halo properties obtained from our XMM-Newton observations. (Note that obs. 0406630201, which yielded $T=11.2 \times 10^{6} \mathrm{~K}$, is not shown in Figure 8(a).)

The X-ray temperatures predicted by the model are in reasonably good agreement with the observed temperatures. However, the emission predicted by this model is clearly too faint: the predicted X-ray EMs are typically an order of magnitude smaller than the observed values. Among the model sightlines that intercept extraplanar SNRs, the median X-ray EM is $1.37_{-0.12}^{+0.09}$ dex smaller than the median observed value of EM $\sin |b|: 0.081 \times 10^{-3}$ versus $\left(1.90_{-0.46}^{+0.41}\right) \times 10^{-3} \mathrm{~cm}^{-6} \mathrm{pc}^{9}{ }^{9}$ As the predicted $\mathrm{X}$-ray temperatures are in reasonable agreement with the observed temperatures, the EM result implies that the $\mathrm{X}$ ray surface brightnesses are also typically underpredicted by an order of magnitude. Note also that our Monte Carlo simulation predicts that about two-thirds of the sightlines would intercept no extraplanar SNRs, whereas we observe hot halo gas on most, if not all, of our sightlines.

The model values in Figures 8(a) and (b) were calculated from the SNR simulations in Shelton (2006) with $E_{0}=0.5 \times 10^{51} \mathrm{erg}$ and $B_{\text {eff }}=2.5 \mu \mathrm{G}$. We find that increasing $E_{0}, B_{\text {eff }}$, or the assumed SN rate all increase the predicted X-ray EMs. Table 4 shows the median X-ray EMs predicted by extraplanar SNR models with different explosion energies, ambient magnetic fields, and SN rates. As can be seen, increasing $B_{\text {eff }}$ has the largest effect on the predicted X-ray EMs. However, it should be noted that the median model values in Table 4 are only for the subset of sightlines that intercept at least one SNR. Increasing $E_{0}$ or the $\mathrm{SN}$ rate increases the fraction of sightlines that intercept at least one SNR, while increasing $B_{\text {eff }}$ decreases that fraction.

\footnotetext{
9 Here and in Section 4.3.2, the errors indicate the $90 \%$ bootstrap confidence interval on the observed median.
}
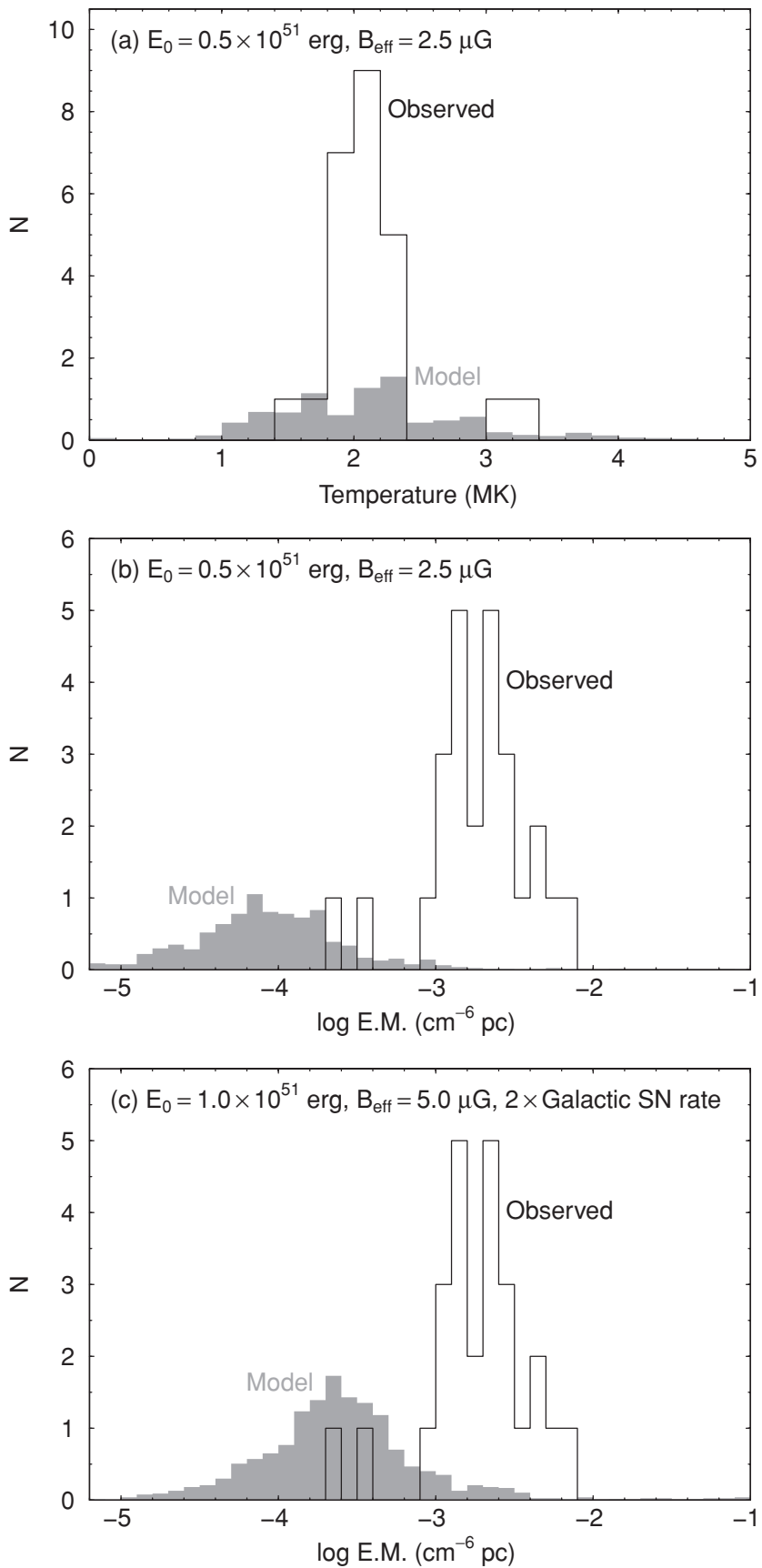

Figure 8. Histograms comparing the halo X-ray temperatures (panel (a)) and $\mathrm{X}$-ray emission measures (panels (b) and (c)) predicted by the extraplanar SNR model (solid gray) with those obtained from our XMM-Newton observations (black outline). The observed emission measures have been multiplied by $\sin |b|$ (see the text for details). The model temperatures and emission measures were derived by characterizing the model spectra with $1 T$ models (see Section 4.2.2). Panels (a) and (b) show model values derived from simulations with $E_{0}=0.5 \times 10^{51} \mathrm{erg}$ and $B_{\text {eff }}=2.5 \mu \mathrm{G}$, with a Galactic SN rate given by Equation (6). Panel (c) shows model emission measures derived from simulations with $E_{0}=1 \times 10^{51} \mathrm{erg}$ and $B_{\text {eff }}=5.0 \mu \mathrm{G}$, with a Galactic SN rate twice as large as that given by Equation (6). The model histograms have been rescaled so they cover the same area as the corresponding observational histograms. Note that $64 \%$ of the model sightlines in panels (a) and (b), and $42 \%$ of the model sightlines in panel (c) intercept no model remnants. Such sightlines have an undefined $\mathrm{X}$-ray temperature and zero $\mathrm{X}$-ray emission measure, and thus do not appear in the above plots.

Figure 8(c) shows the histogram of X-ray EMs predicted by the Shelton (2006) simulations with $E_{0}=1 \times 10^{51} \mathrm{erg}$ and $B_{\text {eff }}=5.0 \mu \mathrm{G}$, with a Galactic SN rate that is twice that 
Table 4

X-ray Emission Measures Predicted by Different Extraplanar SNR Models

\begin{tabular}{lccc}
\hline \hline $\begin{array}{c}E_{0} \\
\left(10^{51} \mathrm{erg}\right)\end{array}$ & $\begin{array}{c}B_{\text {eff }} \\
(\mu \mathrm{G})\end{array}$ & SN Rate $^{\mathrm{a}}$ & $\begin{array}{c}\text { Median EM } \\
\left(10^{-3} \mathrm{~cm}^{-6} \mathrm{pc}\right)\end{array}$ \\
\hline 0.5 & 0 & $1 \times$ & 0.028 \\
0.5 & 2.5 & $1 \times$ & $0.081^{\mathrm{b}}$ \\
0.5 & 5.0 & $1 \times$ & 0.19 \\
1.0 & 5.0 & $1 \times$ & 0.21 \\
1.0 & 5.0 & $2 \times$ & $0.23^{\mathrm{c}}$ \\
Observed & & & $1.90_{-0.46}^{+0.41 \mathrm{~d}}$ \\
\hline
\end{tabular}

Notes. The emission measures were obtained by fitting to the spectra above $0.4 \mathrm{keV}$.

${ }^{a}$ Relative to the rate given by Equation (6).

b Model used in Figures 8(a) and (b).

c Model used in Figure 8(c).

d Median of EM sin $|b|$, with $90 \%$ bootstrap confidence interval.

given by Equation (6) (i.e., the fifth model in Table 4). The histogram of observed values is also plotted for comparison. The model still significantly underpredicts the observed EMs (it underpredicts the median value by $0.91_{-0.12}^{+0.09} \mathrm{dex}$ ). Furthermore, our new Monte Carlo simulation predicts that $\sim 40 \%$ of the sightlines would intercept no extraplanar SNRs-we reiterate that we observe hot halo gas on most, if not all, of our sightlines. We will discuss the results presented here in Section 5.2.2.

\subsection{A Supernova-driven Interstellar Medium}

In this section, we examine another model in which the interstellar gas is heated by SN explosions. This model is distinct from the previous model in a number of ways. The previous model considered only isolated SNRs above $z=130 \mathrm{pc}$, and the X-ray spectra were calculated from one-dimensional hydrodynamical simulations of individual remnants. Here, we use a three-dimensional hyrodynamical simulation of vertically stratified interstellar gas that is heated by discrete $\mathrm{SN}$ explosions (Joung \& Mac Low 2006). Unlike the previous model, some SNe occur in clusters, the evolving SNRs can interact, older remnants may be re-energized by new $\mathrm{SNe}$, and $\mathrm{SNe}$ in the Galactic disk drive a fountain of hot gas up into the halo (Shapiro \& Field 1976; Bregman 1980). However, ionization equilibrium was assumed, and magnetic fields neglected.

\subsubsection{Model Description}

The hydrodynamical simulation used here is described fully in Joung \& Mac Low (2006), and the reader is referred to that paper for more details. The simulation was carried out using Flash, ${ }^{10}$ a parallelized Eulerian hydrodynamical code with adaptive mesh refinement. The simulation box extends from $z=-5 \mathrm{kpc}$ to $z=+5 \mathrm{kpc}$, and has a size of $(0.5 \mathrm{kpc})^{2}$ in the $x y$ plane. The maximum spatial resolution is $1.95 \mathrm{pc}$. The upper and lower boundaries have outflow boundary conditions, while the vertical sides of the simulation box have periodic boundary conditions.

The simulation box was initialized with $1.1 \times 10^{4} \mathrm{~K}$ gas in hydrostatic equilibrium. This gas was then heated by discrete SN explosions, each of which injected $10^{51}$ erg of energy into a small region of the grid. These explosions generally occurred randomly in time and space, with a rate

$$
\begin{aligned}
r(z) & =r_{\mathrm{Ia}}(z)+r_{\mathrm{II}}(z) \\
& =\left(6.2 e^{-|z| / 325 \mathrm{pc}}+167 e^{-|z| / 90 \mathrm{pc}}\right) \mathrm{kpc}^{-3} \mathrm{Myr}^{-1},
\end{aligned}
$$

10 Developed at the University of Chicago Center for Astrophysical Thermonuclear Flashes; http://flash.uchicago.edu/web/.

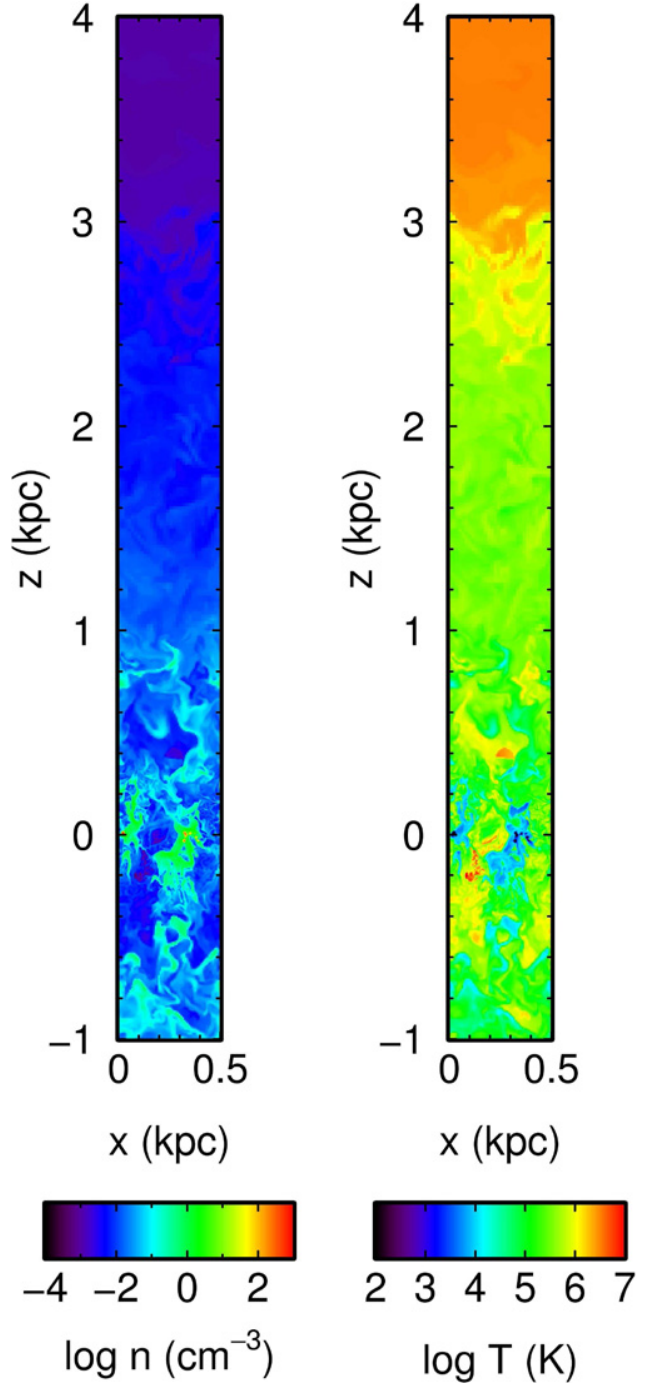

Figure 9. Vertical slices of the density (left) and temperature (right) at $t=120.0 \mathrm{Myr}$, from the SN-driven ISM model of Joung \& Mac Low (2006). Note that the full simulation box extends to $|z|=5 \mathrm{kpc}$.

(A color version of this figure is available in the online journal.)

although $3 / 5$ of the Type II $\mathrm{SNe}$ occurred in clusters of seven to $\approx 40$ explosions. The above rates differ from those in Equation (6) in two ways. First, Joung \& Mac Low (2006) assumed higher Galactic SN rates than Ferrière (1998): $1 / 330 \mathrm{yr}^{-1}$ versus $1 / 445 \mathrm{yr}^{-1}$ (Type I) and $1 / 44 \mathrm{yr}^{-1}$ versus $1 / 52 \mathrm{yr}^{-1}$ (Type II). Second, Equation (6) considers only isolated Type II SNe, whose average height is larger than the average height of all Type II progenitors (266 pc versus 90 pc; Ferrière 1995). The model also includes diffuse heating, due to photoelectric emission from UV-irradiated dust grains, and radiative cooling. Figure 9 shows vertical slices of the density and temperature at $t=120.0 \mathrm{Myr}$.

We calculated X-ray spectra for 242 sightlines looking vertically upward and downward from the Galactic midplane. The viewpoints of these sightlines formed an $11 \times 11$ grid in the $x y$ plane, with a grid spacing of $\approx 49 \mathrm{pc}$. The spectra were calculated using the Raymond \& Smith (1977) spectral code (updated by J. C. Raymond \& B. W. Smith 1993, private communication with R. J. Edgar), assuming that the material along the line of sight is in CIE. We assumed that the gas on the grid is optically thin. We ignored the emission from the first $100 \mathrm{pc}$ of each sightline, as such material is not in 


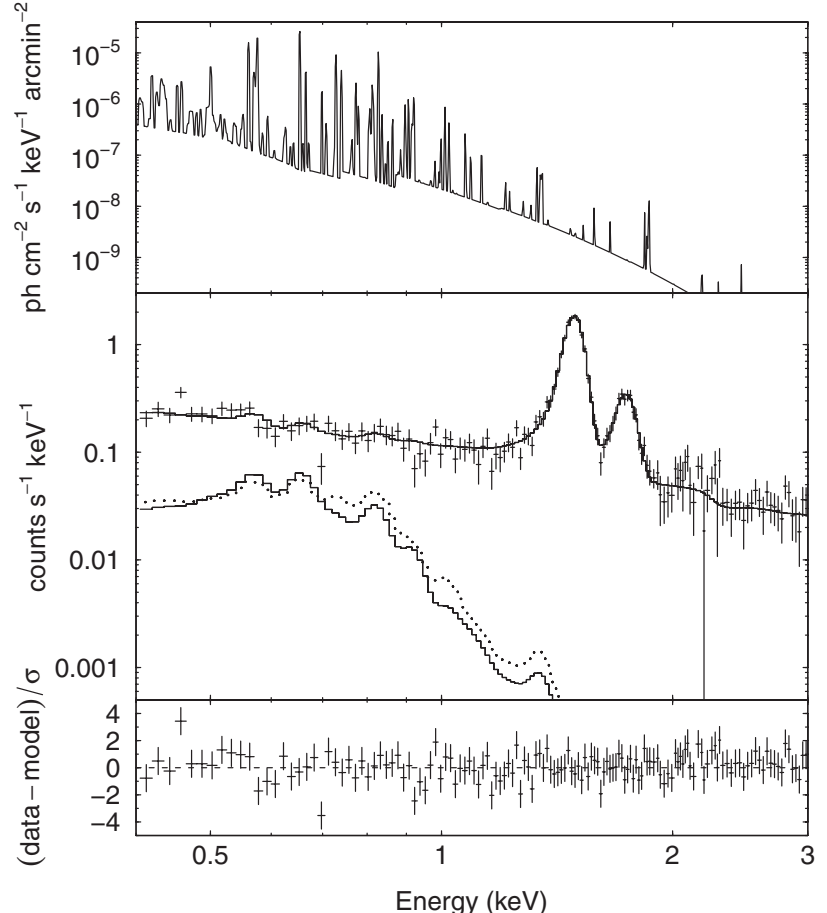

Figure 10. Same as Figure 6, but using the SN-driven ISM model as the input halo model.

the Galactic halo. In our observational analysis, emission from within $\sim 100 \mathrm{pc}$ of the midplane is attributed to our foreground model component, derived from ROSAT shadowing data.

As with the previous model, we used the method described in Section 4.2.2 to characterize the model spectra with $1 T$ models, and used the resulting X-ray temperatures and X-ray EMs to compare with our observations. As in Section 4.2.2, our simulated XMM-Newton spectra included foreground, extragalactic background, and instrumental background components, as well as the halo emission from the SN-driven ISM model. The emission from the halo and extragalactic components was subjected to absorption with $N_{\mathrm{H}}=1.7 \times 10^{20} \mathrm{~cm}^{-2}$. This column density was also used in the subsequent spectral fitting, from which we obtained the characteristic X-ray temperatures and X-ray EMs. Figure 10 shows a simulated XMM-Newton spectrum for one of our model sightlines, along with the best-fitting model. The differential EM for the input halo spectrum is shown in Figure 7 (dashed line). Because we assume that the plasma is in CIE, only plasma with $T \gtrsim 10^{6} \mathrm{~K}$ will contribute in the $X M M$ Newton band. Although the input model predicts emission from a range of temperatures, a model with a $1 T$ halo generally fits the simulated spectra well.

It should be noted that our model spectra do not take into account absorption by cold gas on the grid-when we characterized our model spectra, we assumed that the absorbing column $\left(N_{\mathrm{H}}=1.7 \times 10^{20} \mathrm{~cm}^{-2}\right)$ was located entirely beneath the hot X-ray-emitting gas. For $\sim 2 / 3$ of the model sightlines, the column density of cold gas that is mixed in with the bulk of the $\mathrm{X}$-ray-emitting gas is $<10^{19} \mathrm{~cm}^{-2}$ (i.e., an order of magnitude smaller than the column densities used in our XMM-Newton analysis). We have investigated the effect of ignoring on-grid absorption by creating simulated XMM-Newton spectra from an XSPEC model of the form phabs $*$ (raymond + phabs $*$ raymond), and characterizing the resulting spectra with a model of the form phabs $*$ raymond. As with our previous simulations, the column density of the first phabs component was fixed at
$1.7 \times 10^{20} \mathrm{~cm}^{-2}$. The two raymond components in the input model had the same EM, and temperatures of $1.5 \times 10^{6} \mathrm{~K}$ and $2.5 \times 10^{6} \mathrm{~K}$ (it does not matter which component is the hotter-our conclusion is the same either way). We found that the characteristic X-ray temperatures and X-ray EMs obtained were not strongly affected by the column density between the two raymond components in the input model, at least up to column densities of a few $\times 10^{20} \mathrm{~cm}^{-2}$. We therefore conclude that ignoring on-grid absorption will not adversely affect our results.

In the following, we also compare the predicted X-ray surface brightnesses with our observations. These values were extracted directly from the model spectra.

\subsubsection{Comparing the SN-driven ISM Model with Observations}

Figure 11 shows the time variation of the X-ray spectral properties predicted by the SN-driven ISM model. The model has not settled down to a steady state-the typical X-ray temperature and X-ray surface brightness rise steadily throughout the period shown. However, in this temperature regime, an increase in X-ray surface brightness can be brought about by an increase in temperature as well as by an increase in EM. The typical $\mathrm{X}$-ray EM does not rise steadily throughout the plotted period.

Figure 11 also compares the predicted X-ray properties with the observed values from our XMM-Newton analysis. Similarly to Section 4.2.3, we multiplied our observed EMs and surface brightnesses by $\sin |b|$ before comparing them with the model values. At the earlier times, the model halo is too cool and too faint in the $0.4-2.0 \mathrm{keV}$ band. Around $t=110 \mathrm{Myr}$, the predicted X-ray temperatures are in reasonable agreement with the observed value, but the halo is typically a factor of $\sim 4$ too faint. At later times the model halo is too hot; by $t=155 \mathrm{Myr}$ it is also somewhat brighter than is observed, although the median predicted surface brightness is within $50 \%$ of the observed median.

From Figure 11 it is not clear what state the halo is tending toward. de Avillez \& Breitschwerdt (2004) estimated that a steady state halo in a simulation such as this should be reached in $\lesssim 180$ Myr. However, it is possible that, instead of settling down to a steady state that is hotter and brighter than the observed halo, the variation in X-ray temperature and surface brightness is part of a slow oscillation about a mean state, with a period of at least $\sim 130$ Myr. Determining the final state predicted by this model would probably require running it for at least several more tens of megayears, which is not currently practical. We therefore try two different approaches in comparing the simulation data to our observations.

Our first approach is to assume that the data shown in Figure 11 represent roughly half a cycle in the oscillation of the halo about a mean state. The simulation models only a narrow column of the halo. As our observation directions are not all toward the Galactic poles, our sightlines sample different spatial locations in the halo, which, in a statistical sense, should correspond to different times throughout the cycle. We therefore proceed by averaging the spectra for each sightline from seven of the eight time steps shown in Figure 11 (we do not use the data from $t=118$ Myr, so as not to oversample the times around $t \sim 120 \mathrm{Myr}$ ). We characterize the resulting averaged spectra with $1 T$ models, using the procedure described previously. The results are compared with our observations in Figure 11 (the rightmost data point, labeled "Ave"), and in Figures 12(a) and (b).

Our second approach is to assume that the final time in Figure 11 ( $t=155 \mathrm{Myr}$ ) gives our best estimate of the steady 

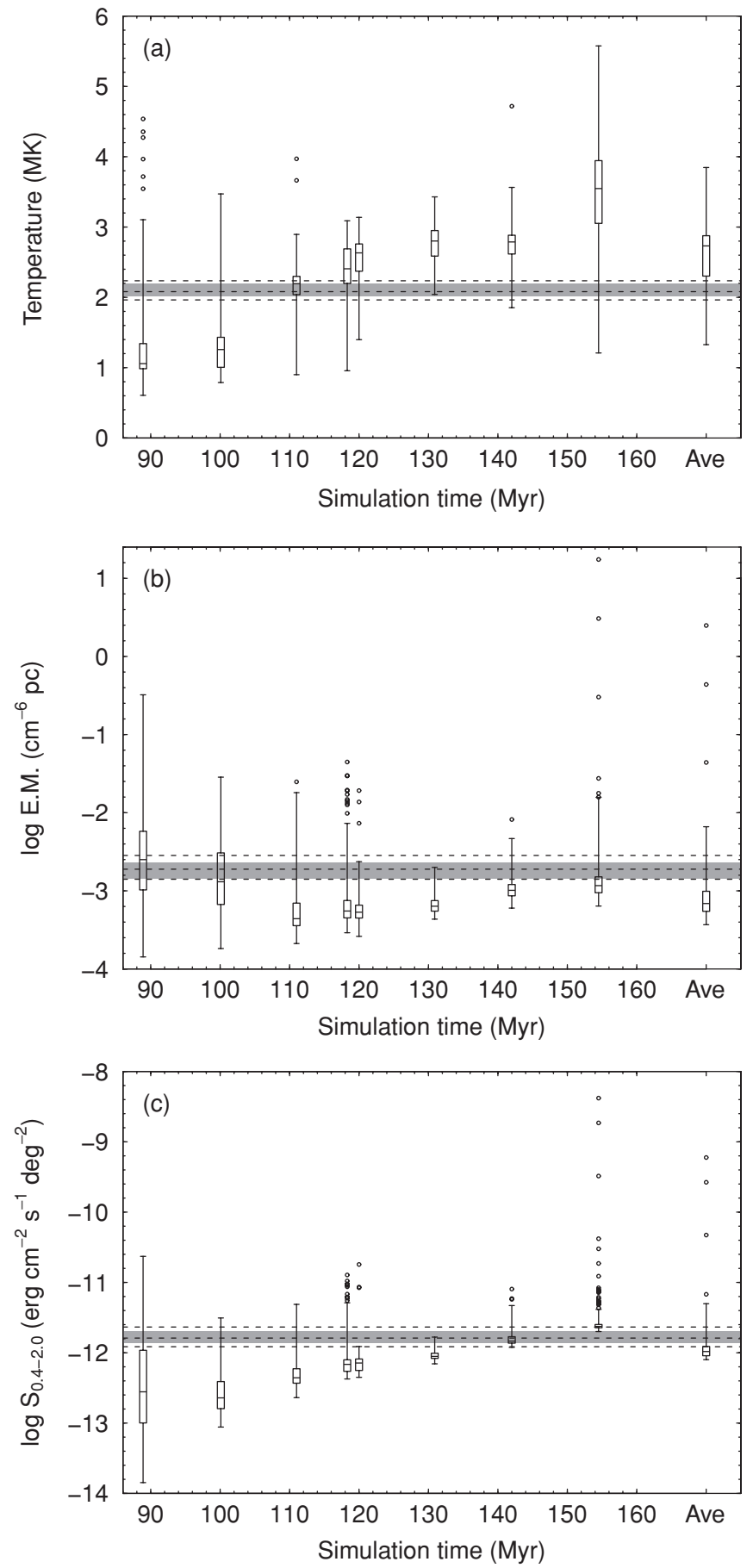

Figure 11. Comparison of the halo (a) X-ray temperatures, (b) X-ray emission measures, and (c) intrinsic $0.4-2.0 \mathrm{keV}$ surface brightnesses predicted by the $\mathrm{SN}$ driven ISM model with the values obtained from our XMM-Newton observations. The boxplots show the predicted values for a range of simulation times. The rightmost data point shows the results obtained by averaging the spectra from several of the time steps (see the text for details). Each box indicates the median and quartiles, and the whiskers extend to the most extreme point that is no more than five times the interquartile range from the box; any outliers are plotted individually with open circles. The horizontal dashed lines indicate the observed median and quartiles, and the gray band indicates the $90 \%$ bootstrap confidence interval on the observed median.

state that the halo is approaching. We use the predictions from that final time in Figures 12(c) and (d).

Whether we use the averaged spectra or the final time step, the predicted halo temperature is too high. The median pre- dicted temperatures are $2.73 \times 10^{6} \mathrm{~K}$ (averaged spectra) and $3.55 \times 10^{6} \mathrm{~K}$ (final time step), against an observed median of $\left(2.08_{-0.07}^{+0.11}\right) \times 10^{6} \mathrm{~K}$. The averaged spectra underpredict the halo EM, although the discrepancy is not as large as with the extraplanar SNR model. The median predicted X-ray EM is $0.44_{-0.12}^{+0.09}$ dex smaller than the median observed value of EM $\sin |b|: 0.69 \times 10^{-3} \mathrm{~cm}^{-6}$ pc versus $\left(1.90_{-0.46}^{+0.42}\right) \times$ $10^{-3} \mathrm{~cm}^{-6} \mathrm{pc}$. For comparison, the discrepancy for the extraplanar SNR model is $1.37_{-0.12}^{+0.09}$ dex. For the spectra from the final time step, the median predicted X-ray EM $\left(1.17 \times 10^{-3} \mathrm{~cm}^{-6} \mathrm{pc}\right)$ is in better agreement with the observed median (the discrepancy is $0.21_{-0.12}^{+0.09}$ dex). The predicted $0.4-2.0 \mathrm{keV}$ surface brightnesses are generally in reasonably good agreement with the observed values, although there are a few sightlines in the final time step of the model that exhibit much greater surface brightnesses. The median predicted values are within $50 \%$ of the median observed value of $S_{0.4-2.0} \sin |b|: 1.04 \times 10^{-12}$ (averaged spectra) and $2.37 \times 10^{-12}$ (final time step), versus $\left(1.62_{-0.05}^{+0.30}\right) \times$ $10^{-12} \mathrm{erg} \mathrm{cm}^{-2} \mathrm{~s}^{-1} \mathrm{deg}^{-2}$ (observed). We will discuss the results presented here in Section 5.2.3.

\section{DISCUSSION}

\subsection{The Temperature and Emission Measure of the Galactic Halo}

In Section 5.2, we will discuss the various physical models of the hot halo that we examined in the previous section. Here, we discuss our XMM-Newton halo measurements, and compare the results with those from other recent studies.

Our measured halo temperatures are typically $\sim 2 \times 10^{6} \mathrm{~K}-$ the median temperature is $2.08 \times 10^{6} \mathrm{~K}$, and the lower and upper quartiles are $1.96 \times 10^{6}$ and $2.24 \times 10^{6} \mathrm{~K}$, respectively. The halo temperatures measured here, using a thermal plasma model, are more tightly constrained than those inferred from the $\mathrm{O}$ VII/O VIII intensity ratio (Paper I). This is partly because fitting with a thermal plasma model uses more of the information in an observed spectrum, and partly because the errors on the intensities of individual lines are not combined in the temperature uncertainty.

Figure 13(a) compares our halo temperatures with various other measurements made with ROSAT, XMM-Newton, and Suzaku (Kuntz \& Snowden 2000; Smith et al. 2007; Galeazzi et al. 2007; Yoshino et al. 2009; Lei et al. 2009; Gupta et al. 2009). For papers with multiple results, the figure caption indicates the specific results that we have plotted. Our temperatures are typically lower than the temperature obtained by Kuntz \& Snowden (2000), using ROSAT All-Sky Survey data. The most likely reason for this is that Kuntz \& Snowden (2000) used a two-temperature $(2 T)$ model for the halo, whereas as we used a $1 T$ model. An additional, lower-temperature halo component could account for some of the lower-energy flux in the XMMNewton band, enabling the other component to shift toward higher temperatures. However, in practice we found that we were unable to constrain a $2 T$ model using our XMM-Newton data, as XMM-Newton's sensitivity does not extend to as low energies as ROSAT's. A $1 T$ halo model adequately described our XMM-Newton spectra.

Our halo temperatures are generally in good agreement with those measured by Smith et al. (2007), Galeazzi et al. (2007), Gupta et al. (2009), Lei et al. (2009), and Yoshino et al. (2009). All of these measurements are from $1 T$ halo models. Note that the Yoshino et al. (2009) temperatures were obtained with a 

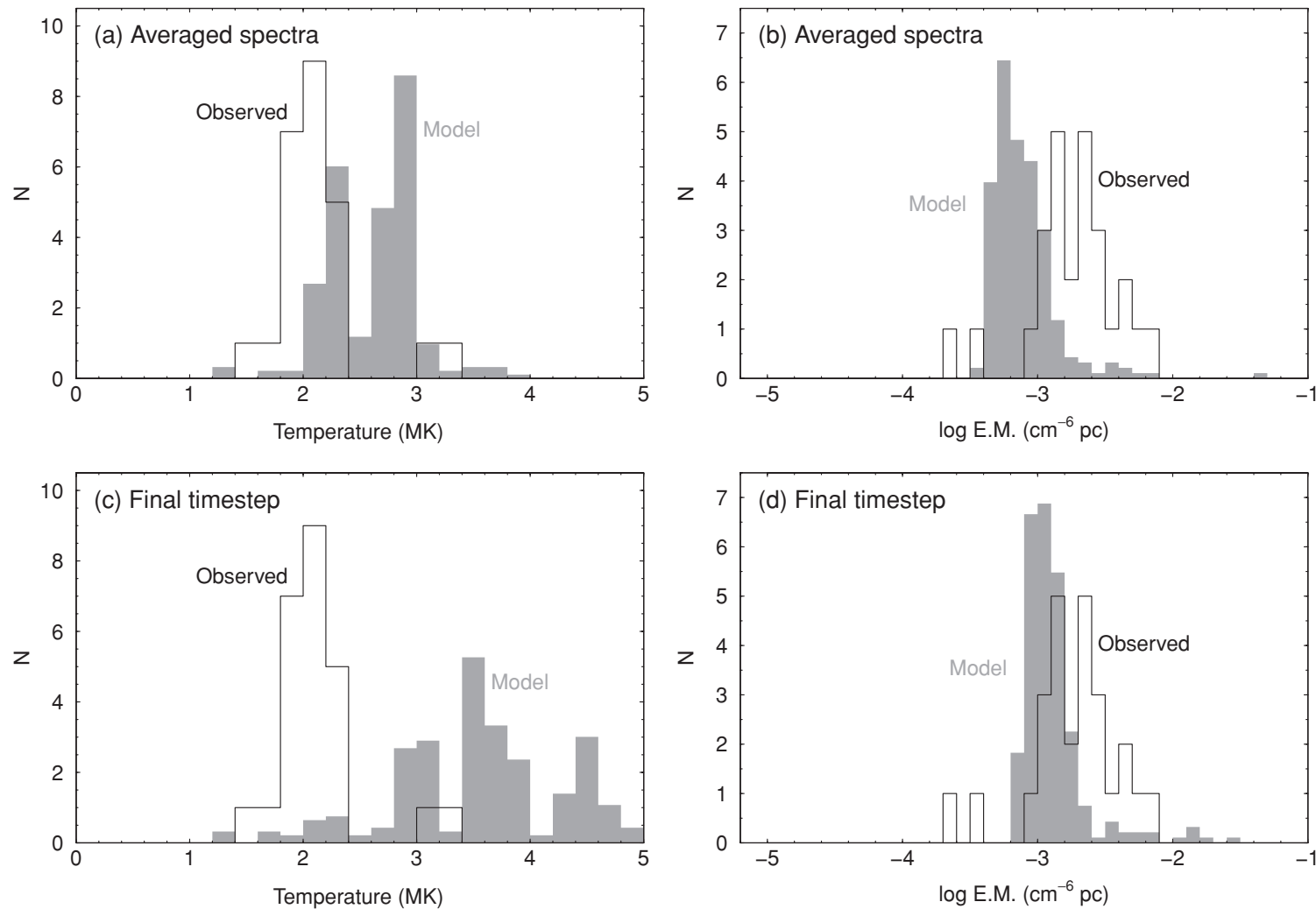

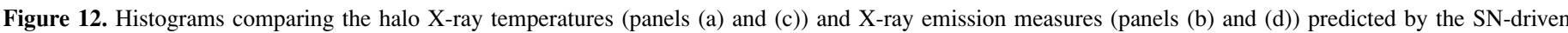

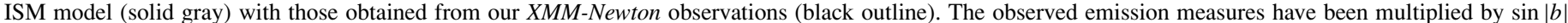

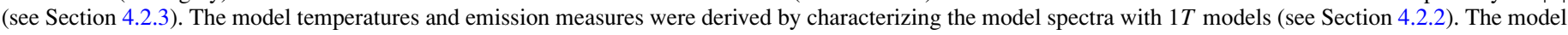

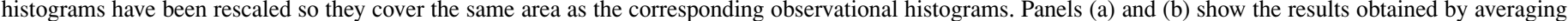

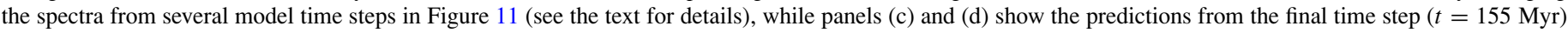
in Figure 11.

fixed foreground model for all their sightlines, and $\mathrm{Fe}$ and $\mathrm{Ne}$ abundances (relative to $\mathrm{O}$ ) that were free to vary (their Table 6). When they fitted their model with an independent foreground model and fixed abundances for each sightline, Yoshino et al. obtained systematically higher halo temperatures (their Tables 3 and 4). However, Yoshino et al. argue that these higher temperatures are mostly determined by the $\mathrm{Fe}-\mathrm{L}$ and $\mathrm{Ne}-$ $\mathrm{K}$ emission, and are inconsistent with the behavior of the $\mathrm{O}$ VII and $\mathrm{O}$ vIII emission. When they measured the halo temperatures that we have used in Figure 13(a), Yoshino et al. found that about half of their sightlines required either an overabundance of $\mathrm{Fe}$ and $\mathrm{Ne}$, or an additional hotter emission component.

The halo temperatures measured here and in other recent studies are fairly constant across the sky. This is most likely because gas with $T \lesssim 1 \times 10^{6} \mathrm{~K}$ would be difficult to detect with $X M M-N e w t o n$ (unless it has a large EM), while gas with $T \gtrsim 3 \times 10^{6} \mathrm{~K}$ would escape from the Galactic potential well (e.g., Bregman 2009).

Figure 13(b) compares our halo EMs with those from the studies discussed above. In general, our values are in good agreement with the values from the other studies. The large EM obtained by Lei et al. (2009) may be partly due to their using Wilms et al. (2000) abundances. The Wilms et al. (2000) oxygen abundance is a factor of 1.8 smaller than the Anders \& Grevesse (1989) value. As oxygen emission tends to dominate the halo X-ray spectrum, a smaller oxygen abundance will result in a larger X-ray EM.

There is considerable scatter in the halo EM, with the values spanning an order of magnitude $\left(\sim 0.0005-0.006 \mathrm{~cm}^{-6} \mathrm{pc}\right)$. This patchiness to the halo has already been pointed out by Yoshino et al. (2009) and in Paper I. We will discuss in more detail the various physical models that we have examined in Section 5.2, but we note here that a patchy halo favors an inhomogeneous, stochastic heat source, such as $\mathrm{SNe}$, as opposed to accretion of extragalactic material, which we would expect to be fairly homogeneous.

\subsection{Physical Models of the Hot Halo}

Here, we discuss each of the models presented in Section 4 in turn.

\subsubsection{The Disk Galaxy Formation Model}

In Section 4.1, we examined a disk galaxy formation model (Toft et al. 2002; Rasmussen et al. 2009), which predicts the existence of a hot halo extended over tens of kiloparsecs, formed from material falling into the galaxy's potential well. Using results from Rasmussen et al. (2009), we find that the emissionweighted mean temperature predicted for a Milky-Way-sized galaxy $\left(v_{\mathrm{c}} \sim 220 \mathrm{~km} \mathrm{~s}^{-1}\right)$ is in good agreement with the median observed halo temperature, but the model underpredicts the $\mathrm{X}$-ray luminosity of the halo by at least an order of magnitude.

The above results suggest that the extended hot halo predicted by disk galaxy formation models is not a major contributor to the halo X-ray emission that we observe with XMM-Newton. However, this interpretation is complicated by the simulations of Crain et al. (2010). They argue that the stellar feedback in the 

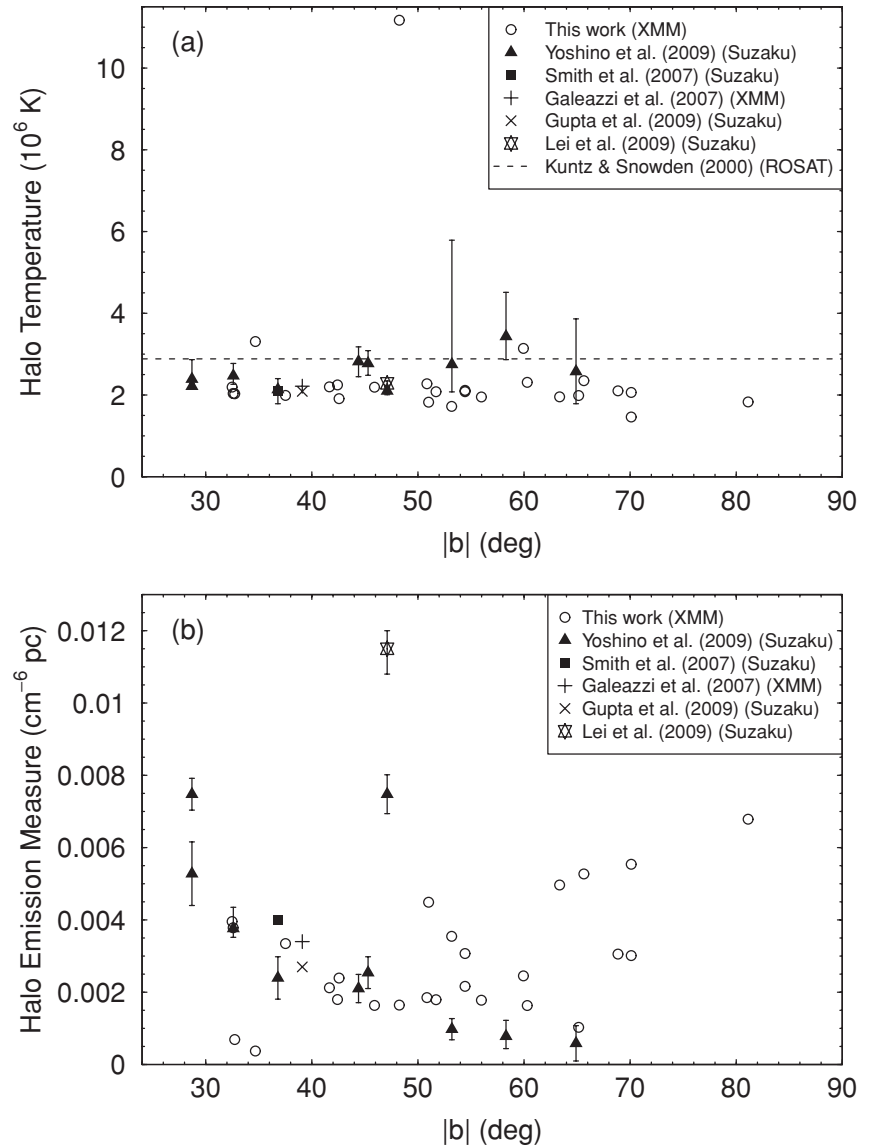

Figure 13. Halo (a) temperatures and (b) emission measures plotted against Galactic latitude. The open circles show the results from this work, with the error bars omitted for clarity. The solid triangles show the results from Yoshino et al. (2009) with $|b|>20^{\circ}$; specifically, the results obtained with a fixed foreground model for all sightlines (their Table 6). The solid square shows the results from a Suzaku shadowing observation of MBM 12 (Smith et al. 2007). The "+" and the " $\times$ " show the results from shadowing observations of MBM 20, carried out with XMM-Newton and Suzaku, respectively (Galeazzi et al. 2007; Gupta et al. 2009). Specifically, the XMM-Newton result is the result obtained with frozen abundances from Table 2 in Galeazzi et al. (2007), and the Suzaku result is from row 3 of Table 2 in Gupta et al. (2009). The star shows the results from a Suzaku shadowing observation of a dusty filament in the southern Galactic hemisphere (Lei et al. 2009), specifically, Model 2 from their Table 1. The horizontal dashed line shows the temperature derived by Kuntz \& Snowden (2000) from the ROSAT All-Sky Survey.

Rasmussen et al. (2009) simulations is too weak, resulting in too much mass ending up in stars and too little mass in hot gas. Ideally, we would compare the surface brightness of the accreted extragalactic material in the Crain et al. (2010) simulations with the halo surface brightness obtained from our XMM-Newton observations, but this is not possible for two reasons. First, in addition to infalling extragalactic material, the Crain et al. model includes interstellar gas that was heated by stars and SNe and transferred upward by an outflow (similar to the galactic fountain in the model described in Section 4.3). Second, their model predictions are in terms of luminosity rather than surface brightness. Because gas heated by stars and SNe will tend to be concentrated nearer the disk, compared with the more extended halo of accreted extragalactic material, it may provide a much larger surface brightness per unit luminosity than the extended halo gas. Predictions of the separate contributions to the surface brightness due to the gas heated by stellar processes and the extended halo are not currently available from the Crain et al. model. Such predictions are needed to determine whether or not accreted extragalactic material makes a major contribution to the observed halo X-ray emission.

Although surface brightness predictions are not currently available, we can make rough comparisons between the total X-ray luminosity predicted by Crain et al. (2010) and the halo luminosity expected from the XMM-Newton data. An $L_{\mathrm{X}}-T$ plot derived from their simulations shows that galaxies with emission-weighted halo temperatures of $\sim 2 \times 10^{6} \mathrm{~K}$ have $0.3-2.0 \mathrm{keV}$ luminosities of $\sim(3-40) \times 10^{39} \mathrm{erg} \mathrm{s}^{-1}$ (R. Crain 2010, private communication), compared with $<0.2 \times$ $10^{39} \mathrm{erg} \mathrm{s}^{-1}$ from Rasmussen et al. (2009). In Section 4.1, we derived a Galactic halo luminosity of $1.9 \times 10^{39} \mathrm{erg} \mathrm{s}^{-1}$ from our observations, which is slightly smaller than the range of luminosities predicted by Crain et al. (2010). However, this observed luminosity was calculated assuming that the observed emission came from a uniform sphere of radius $R_{\mathrm{sph}}=15 \mathrm{kpc}$. Figure 2 in Crain et al. (2010) implies that the emission in their model is much more extended than this, with $R_{\mathrm{sph}} \sim 50 \mathrm{kpc}$. Using this larger radius leads to an observed halo luminosity of $1.3 \times 10^{40} \mathrm{erg} \mathrm{s}^{-1}$, which lies within the range predicted by Crain et al.

\subsubsection{The Extraplanar SNR Model}

In Section 4.2, we examined a model in which the hot halo gas is contained in an ensemble of isolated extraplanar SNRs (Shelton 2006). The X-ray temperatures predicted by this model are in good agreement with the observed values. However, the predicted X-ray EMs, obtained from model spectra above $0.4 \mathrm{keV}$, are typically an order of magnitude smaller than the observed values. We can increase the predicted X-ray EMs by increasing the $\mathrm{SN}$ explosion energy, $E_{0}$, the effective ambient magnetic field, $B_{\text {eff }}$, or the $\mathrm{SN}$ rate. However, doubling all three of these parameters still resulted in X-ray EMs that were too small.

Of the above three parameters, $B_{\text {eff }}$ has the largest effect on the predicted X-ray EMs (see Table 4 ). Increasing $B_{\text {eff }}$ increases the non-thermal pressure, and therefore increases the compression of the hot X-ray-producing bubble. This increased compression increases the density and temperature of the bubble, increasing the X-ray EM inferred from the predicted emission in the $X M M-N e w t o n$ band. We have not carried out simulations with $B_{\text {eff }}>5.0 \mu \mathrm{G}$, but if we extrapolate the values in Table 4 , we find we would need a $B_{\text {eff }}$ of a few tens of microgauss to match the observed EMs. Such a magnetic field implies a non-thermal pressure $P_{\mathrm{nt}} / k \gtrsim 10^{5} \mathrm{~cm}^{-3} \mathrm{~K}$. This is an implausibly high non-thermal pressure for the halo, as it is several times larger than the midplane value (Boulares \& Cox 1990; Ferrière 2001). In addition, the model with $B_{\text {eff }}=2.5 \mu \mathrm{G}$ predicts that hot gas would be seen on only $\sim 1 / 3$ of sightlines. As noted above, increasing $B_{\text {eff }}$ results in smaller, hotter remnants. In addition, these smaller remnants are brighter and so shorter lived. As a result, increasing $B_{\text {eff }}$ means that even fewer sightlines would intercept remnants. In reality, we find hot gas on most, if not all, of our XMM-Newton sightlines. Therefore, we cannot bring the extraplanar SNR model into agreement with our observations by increasing the assumed effective ambient magnetic field.

Increasing the $\mathrm{SN}$ rate increases the number of SNRs expected to lie along a given sightline, and so increases the predicted Xray EMs. However, we would have to increase the $\mathrm{SN}$ rate given by Equation (6) by a factor of $\sim 6$ in order to give the same increase in the median X-ray EM that we see when we increase $B_{\text {eff }}$ from 2.5 to $5.0 \mu \mathrm{G}$. To match the observations, we would have to increase the $\mathrm{SN}$ rate by an even larger factor. 
The Galactic SN rate of $\sim 2$ per century is constrained to within a factor of $\sim 2$ (see the online Supplementary Information ${ }^{11}$ for Diehl et al. 2006). If the SN scale heights in Equation (6) are well constrained, the halo SN rate is also constrained to within a factor of $\sim 2$. Even if we double the $\mathrm{SN}$ scale heights in Equation (6), the integrated SN rate above $|z|=130 \mathrm{pc}$ only increases by a factor of 2.5. Therefore, to bring the extraplanar SNR model into agreement with our observations we would have to increase the SN rate by an unrealistic amount. We also cannot bring the model into agreement with our observations by increasing $E_{0}$, as Table 4 shows that the median X-ray EM depends only weakly on $E_{0}$.

We therefore conclude that the hot halo gas that we observe with XMM-Newton is not primarily due to an ensemble of isolated extraplanar SNRs. Most of this population of remnants would be relatively old (age $>1 \mathrm{Myr}$ ) and faint in the $X M M$ Newton band. It should be noted, however, that this result does not imply that extraplanar SNRs do not contribute to the hot halo gas at all. In the XMM-Newton band (above $0.4 \mathrm{keV}$ ), their contribution is masked by brighter emission from an $\mathrm{SN}$ driven galactic fountain (see Section 5.2.3). At lower energies, extraplanar SNRs could still contribute significantly to the hot gas observed in the $1 / 4 \mathrm{keV}$ band, as originally suggested by Shelton (2006). In addition, young, bright remnants, although rare, do produce emission that is detectable by Suzaku (Henley \& Shelton 2009) and that should also be detectable by XMMNewton.

\subsubsection{The SN-driven ISM Model}

In Section 4.3, we examined a hydrodynamical simulation of vertically stratified interstellar gas, driven by $\mathrm{SN}$ explosions (Joung \& Mac Low 2006). Unlike the extraplanar SNR model, the SNRs do not evolve in isolation, and the model results in a galactic fountain of hot gas up into the halo (Shapiro \& Field 1976; Bregman 1980). Also, for this model we assumed that the gas is in CIE, whereas the extraplanar SNR model includes self-consistent modeling of the ionization evolution.

As with the extraplanar SNR model, we folded the model spectra through the XMM-Newton response, added photon noise, and characterized the resulting spectra with $1 T$ models. Before going on to discuss the comparison of the resulting X-ray temperatures and EMs with our XMM-Newton observations, we shall look at how well these X-ray spectral properties reflect the properties of the gas on the hydrodynamical grid.

Figure 14(a) compares the X-ray temperatures derived from the $1 T$ models with the mean temperature along each model sightline through the hydrodynamical grid, weighted by the 0.4-2.0 keV emission. We find that the X-ray temperatures are in reasonable agreement with the emission-weighted mean temperatures (typically within $0.4 \times 10^{6} \mathrm{~K}$ ). Figure 14(b) compares the X-ray EM for each sightline with the EM of the gas with $T>10^{6} \mathrm{~K}$ along that sightline (we use this quantity because there is no obvious analog to the emission-weighted mean temperature). The X-ray EM underestimates the EM of gas with $T>10^{6} \mathrm{~K}$, although the two quantities are well correlated and generally agree within a factor of 2.5 . Overall, we conclude that the properties derived from the $1 T$ models are good characterizations of the hot gas on the hydrodynamical grid

\footnotetext{
11 http://www.nature.com/nature/journal/v439/n7072/suppinfo/ nature04364.html
}
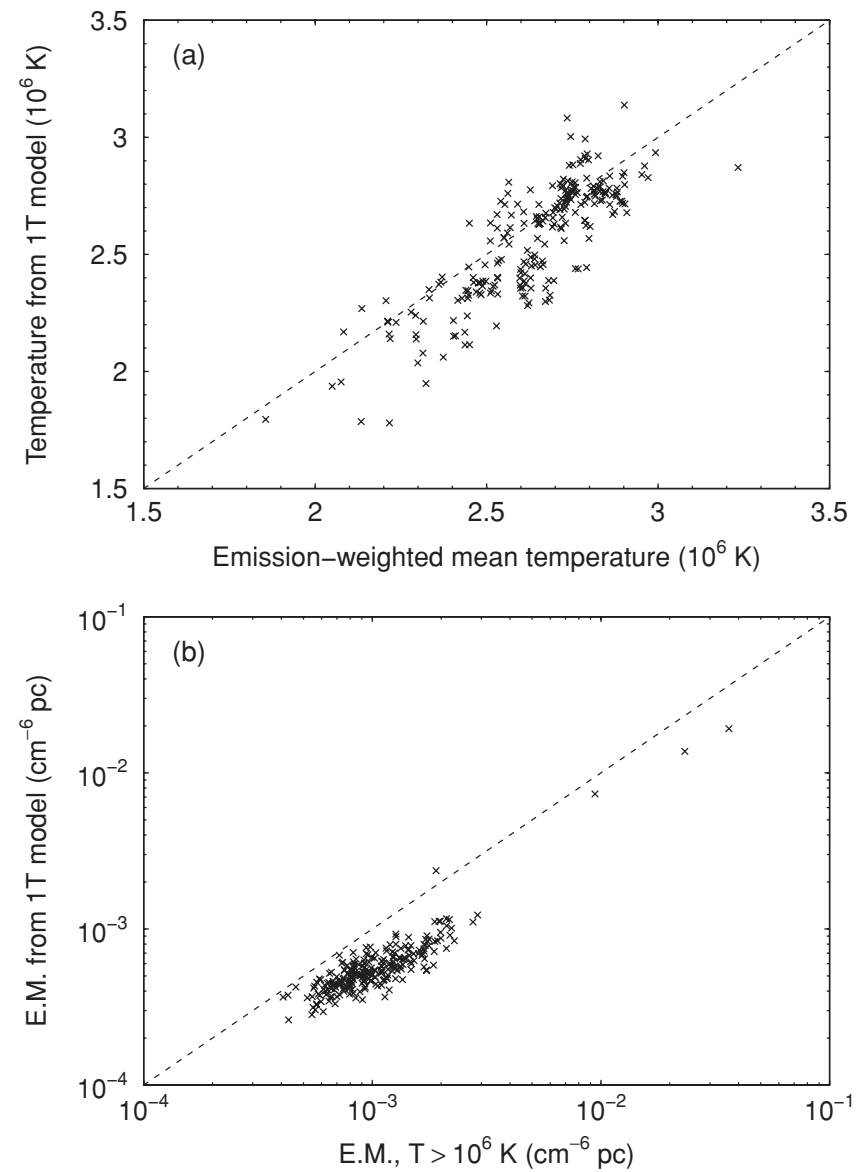

Figure 14. Comparison of X-ray spectral properties derived from $1 T$ models (ordinates) and properties derived directly from the hydrodynamical data (abscissae) for each sightline through the SN-driven ISM model at $t=120 \mathrm{Myr}$.

The $0.4-2.0 \mathrm{keV}$ halo surface brightness predicted by this model is in good agreement with the observations (within $\sim 50 \%$ ). As the emission predicted by this model is much brighter, it will mask the contribution from extraplanar SNRs. Note that this model does include some in situ heating by extraplanar SNe. The integrated halo SN rate above $|z| \sim 100 \mathrm{pc}$ is $\sim 2$ times larger in this model than in the extraplanar SNR model (although the volumetric SN rate falls off more rapidly with $|z|$ in this model). However, this larger integrated SN rate is not enough to explain the differences in X-ray surface brightness between the models: this model predicts emission an order of magnitude brighter than the extraplanar SNR model. The majority of the halo X-ray emission in this model comes from hot gas that is driven from the disk into the halo by a galactic fountain. As mentioned in Section 5.2.1, it is possible that the emission from a galactic outflow or fountain also masks the contribution from an extended hot halo of accreted material.

Although the median predicted X-ray surface brightness and $\mathrm{X}$-ray EM are in good agreement with the observed medians, the halo X-ray temperature predicted by this model is $\sim 1 \times 10^{6} \mathrm{~K}$ larger than our measured value, independent of whether the simulated halo is undergoing a long-period $(P \gtrsim 100 \mathrm{Myr})$ oscillation about some mean state, or is settling down to a steady state. Given the uncertainties in the model predictions (due to the time variability shown in Figure 11), these discrepancies are probably insufficient to rule out this model. Nevertheless, we discuss below three possible causes for the temperature discrepancy: (1) the simulation overpredicts the temperature 
of the hot gas in the halo, (2) the temperature of the hot gas does not accurately predict the X-ray spectrum, because the gas is not in ionization equilibrium, or (3) a bias in our spectral analysis causes us to underestimate the observed halo X-ray temperature. Correcting for whichever of these effects turn out to be important may also affect the X-ray surface brightnesses and EMs. However, it is not easy to foresee whether these corrections will increase or decrease the discrepancies between the predicted and observed surface brightnesses and EMs.

The first possible cause of the temperature discrepancy is that the simulation overpredicts the temperature of the hot gas in the halo. The simulation does not include thermal conduction, which could in principle lower the temperature of the hot X-ray-emitting gas, although thermal conduction in the ISM can be suppressed by magnetic fields. de Avillez $\&$ Breitschwerdt (2004) argue that turbulent diffusion is more efficient at mixing hot and cold gas than thermal conduction. However, the efficiency of this mixing will be underestimated if the mixing is not fully resolved. In the simulation used here, the spatial resolution is between $1.95 \mathrm{pc}$ and $15.6 \mathrm{pc}$, but the adaptive mesh refinement criterion is chosen to focus maximum numerical resolution on the $200 \mathrm{pc}$ above and below the midplane, so the gas is less well resolved in the halo than in the disk. As a result, it is possible that turbulent mixing of hot and cold gas is underresolved in the halo, resulting in an overestimate of the temperature of the X-ray-emitting gas (Fujita et al. 2009).

A lack of spatial resolution may also suppress the radiative cooling of the hot gas-averaging the hot gas density over large cells eliminates local denser regions that would radiate more efficiently (de Avillez \& Breitschwerdt 2004). de Avillez \& Breitschwerdt (2004) found that the filling factor of hot $\left(T>10^{5.5} \mathrm{~K}\right)$ gas was not significantly affected by the spatial resolution of their simulations. However, there is insufficient information to determine how the emission-weighted mean temperature of the X-ray-emitting gas would be affected. Simulations with higher spatial resolution in the halo will help determine whether or not the mixing of hot and cold gas and the radiative cooling of hot gas are adequately resolved for predicting the X-ray emission.

Joung \& Mac Low (2006) pointed out that the average gas density at several disk scale heights and beyond $(0.2 \mathrm{kpc} \lesssim|z| \lesssim$ $2.5 \mathrm{kpc}$ ) is somewhat underpredicted in their model compared to observations. They suggested that additional components of pressure from the magnetic field and cosmic rays may contribute significantly to the support in the vertical direction (see Section 3.1 of their paper). Additional vertical pressure support will lead to larger disk scale heights and hence larger gas masses and lower temperatures at 1-2 kpc heights, which will reduce the discrepancy between the observations and the SN-driven ISM model. Preliminary results from magnetohydrodynamics simulations are in agreement with this expectation (A. Hill et al. 2010, in preparation).

The second possible cause for the discrepancy between the predicted and observed X-ray temperatures is that the hot gas is out of equilibrium. This gas was shock-heated by $\mathrm{SNe}$ - this rapid heating causes the ionization temperature (which determines the X-ray spectrum) to lag behind the kinetic temperature (which is the quantity obtained from the hydrodynamical simulation). This ionization evolution was followed self-consistently in the one-dimensional SNR models described in Section 4.2, but not in this three-dimensional simulation. CIE is reached on a timescale of $t_{\mathrm{eq}} \sim 10^{12} n_{\mathrm{e}}^{-1} \mathrm{~s}$, where $n_{\mathrm{e}}$ is the electron density in $\mathrm{cm}^{-3}$ (Masai 1994). In the simulation, the density of gas with $T>10^{6} \mathrm{~K}$ is $\lesssim 10^{-3} \mathrm{~cm}^{-3}$, implying $t_{\mathrm{eq}} \gtrsim 30 \mathrm{Myr}$. This is similar to the dynamical timescale $\left(5 \mathrm{kpc} / c_{\mathrm{s}} \sim 30 \mathrm{Myr}\right.$, where $c_{\mathrm{s}}$ is the sound speed in $10^{6} \mathrm{~K}$ gas), and so we would expect the hot gas to be at least partially underionized.

Calculating the X-ray spectrum of an underionized plasma requires knowledge of the ionization balance, which in turn depends on the history of the plasma. However, we note that an underionized plasma will have fewer higher-stage ions (e.g., $\mathrm{O}^{+7}$ ) relative to lower-stage ions (e.g., $\mathrm{O}^{+6}$ ) than we would expect from the kinetic temperature. Therefore, if the halo is underionized, modeling the observed emission with a CIE model will underestimate the kinetic temperature of the halo gas. We have confirmed this by simulating XMM-Newton observations of an underionized plasma (using the XSPEC nei model), and characterizing the resulting simulated spectra with $1 T$ CIE models (for simplicity, here we just use the nei model as an input for our simulations, instead of the full multicomponent model used in Section 4.2.2). For $n_{\mathrm{e}} t \lesssim 10^{9} \mathrm{~cm}^{-3} \mathrm{~s}$, CIE models do not fit the simulated spectra well (these models underestimate the low-energy flux). However, for $n_{\mathrm{e}} t \sim 10^{9}-10^{12} \mathrm{~cm}^{-3} \mathrm{~s}, 1 T$ CIE models give good fits to the simulated spectra, but underestimate the input kinetic temperature.

The above discussion considers only the hot gas being underionized. An additional possible source of X-rays is delayed recombination from overionized gas-gas that has undergone rapid adiabatic cooling as it expands into the halo (Breitschwerdt \& Schmutzler 1994, 1999). With our current simulation data, it is difficult to estimate the contribution of delayed recombination to the halo emission, relative to the emission from the hot gas. Simulations that can track the ionization evolution of the plasma are needed to determine the extent to which the assumption of $\mathrm{CIE}$ affects the predicted spectra, and hence the derived X-ray temperature.

The third possible cause of the temperature discrepancy is that something (other than the assumption of CIE) is biasing the temperatures measured from our XMM-Newton observations. The discrepancy is not because we characterize the halo emission with a $1 T$ model, as opposed to a $2 T$ model (e.g., Kuntz $\&$ Snowden 2000; see Section 5.1), because we characterize the model spectra in the same way. If we are underestimating the halo temperature, the most likely cause is that our foreground (LB and/or SWCX) model is too faint; in particular, that it underestimates the foreground $\mathrm{O}$ VII emission (underestimating the foreground $\mathrm{O}$ VII emission means that the halo $\mathrm{O}$ VII emission is overestimated relative to the halo $\mathrm{O}$ VIII emission, causing the measured halo temperature to shift to a lower value). However, our halo temperatures are in good agreement with those from other recent studies (see Section 5.1), which used different methods to estimate the foreground emission. It therefore seems unlikely that a bias in the halo temperature measurements is causing the discrepancy between the observed temperatures and those predicted by the SN-driven ISM model. Nevertheless, an accurate model of SWCX emission will help evaluate our method for estimating the foreground emission.

\section{SUMMARY AND CONCLUSIONS}

We have analyzed 26 high-latitude XMM-Newton observations of the SXRB, concentrating in particular on the emission from the Galactic halo. These observations were chosen from a 
much larger set of observations (Paper I) as they are expected to be the least contaminated by SWCX emission. We modeled the 0.4-5.0 keV X-ray spectra with emission components from the foreground, the Galactic halo, and the extragalactic background, with additional components modeling parts of the instrumental background. Assuming a single-temperature CIE plasma model for the halo, we typically obtained halo temperatures of $\sim(1.8-2.4) \times 10^{6} \mathrm{~K}$, and EMs of $\sim 0.0005-0.006$ $\mathrm{cm}^{-6} \mathrm{pc}$, in good agreement with previous studies. While the halo temperature is fairly constant, the EM exhibits significant sightline-to-sightline variation.

We compared the observed X-ray properties of the halo with the predictions of three physical models for the origin of the hot halo gas: (1) a disk galaxy formation model, which predicts the existence of a hot halo extended over tens of kiloparsecs (Toft et al. 2002; Rasmussen et al. 2009; Crain et al. 2010); (2) a model in which the halo is heated by extraplanar $\mathrm{SNe}$, and the hot gas resides in isolated SNRs (Shelton 2006); and (3) a more comprehensive model of SN heating of the ISM, in which the SNRs do not evolve in isolation, but drive a fountain of hot gas from the disk into the halo (Joung \& Mac Low 2006).

Model 2 matches the observed halo temperature reasonably well, but this model predicts emission at least an order of magnitude too faint in the XMM-Newton band, implying that another source of hot gas is needed, in addition to isolated extraplanar SNRs. With Model 1, the conclusions are more uncertain: the original simulations that we examined (from Rasmussen et al. 2009) predicted luminosities at least an order of magnitude too faint in the XMM-Newton band, whereas newer simulations with stronger stellar feedback predict larger X-ray luminosities (Crain et al. 2010), in better agreement with those inferred from our observations. However, the predicted emission from that model includes contributions not only from accreted extragalactic material falling into the galactic potential well, but also from material that has been heated by stars and flowed out from the disk. Predictions of the relative X-ray surface brightnesses due to these two processes are needed to determine whether or not emission from accreted extragalactic material is a major contributor to the observed halo X-ray emission.

A flow of hot gas from the disk into the halo, in the form of an SN-driven galactic fountain (Shapiro \& Field 1976; Bregman 1980), also occurs in the SN-driven ISM model (Model 3). The X-ray surface brightness predicted by this model is in good agreement with the observed surface brightness of the halo, although it should be noted that the halo in this model has not yet settled down to a steady state. Therefore, while we cannot currently rule out the possibility of a significant contribution from accreted extragalactic material, our analysis indicates that hot gas in an SN-driven galactic fountain is a major (possibly dominant) contributor to the halo X-ray emission. While previous work has shown that disk SNe produce sufficient energy to heat the halo (e.g., Wang 1998; Shelton et al. 2007; Yao et al. 2009; Yoshino et al. 2009), to the best of our knowledge this is the first time that CCD-resolution spectra of the halo have been compared with predictions from a hydrodynamical model of a galactic fountain.

Although the halo X-ray surface brightness predicted by the SN-driven ISM model is in good agreement with the observations, this model overpredicts the X-ray temperature of the halo. We discussed various possible reasons for this discrepancy, including the simulation underresolving the mixing of hot and cold gas, the simulation omitting important sources of pressure support that would increase the disk scale height and lower the temperature at $|z| \sim 1-2 \mathrm{kpc}$, the hot halo gas being underionized, and our potentially underestimating the foreground $\mathrm{O}$ VII intensity in the observed spectra, which would in turn cause us to underestimate the observed halo temperature (although this final possibility seems unlikely, given the good agreement between our temperature measurements and those from other recent studies). On the modeling side, future simulations that have higher resolution in the halo, that include magnetic fields, and that track the ionization evolution (Benjamin et al. 2001) may help reduce the discrepancy between the predicted and observed X-ray temperature. A SWCX model that can more accurately predict the foreground emission will help on the observational side.

We can further test the current and future simulations with additional observations. We plan to expand our XMM-Newton survey (Paper I) to cover the whole sky. X-ray absorption line measurements can also be used to test the models. The significant sightline-to-sightline variation in the observed halo emission shows that, in order to examine halo models in detail, data from as many sightlines as possible should be used.

We thank Jesper Rasmussen for supplying us with X-ray luminosities and temperatures derived from disk galaxy simulations, and for helpful discussions. We also thank Rob Crain for making us aware of Crain et al. (2010), and for supplying us with predictions from their simulations. We thank the anonymous referee, whose detailed technical comments have improved the data analysis presented in this paper. We acknowledge use of the $\mathrm{R}$ software package (R Development Core Team 2008). This research is based on observations obtained with XMM-Newton, an ESA science mission with instruments and contributions directly funded by ESA Member States and NASA. The software used in this work was in part developed by the DOE-supported ASCI/ Alliance Center for Astrophysical Thermonuclear Flashes at the University of Chicago. The simulations described in Section 4.3 were performed at the Pittsburgh Supercomputing Center and the National Center for Supercomputing Applications supported by the NSF. D.B.H. and R.L.S. acknowledge funding from NASA grant NNX08AJ47G, awarded through the Astrophysics Data Analysis Program. K.J.K. and R.L.S. acknowledge funding from NASA grant NNX09AD13G, awarded through the Astrophysics Theory and Fundamental Physics Program. M.R.J. and M.-M.M.L. acknowledge funding from NASA/SAO grant TM0-11008X.

\section{REFERENCES}

Anders, E., \& Grevesse, N. 1989, Geochim. Cosmochim. Acta, 53, 197

Bałucińska-Church, M., \& McCammon, D. 1992, ApJ, 400, 699

Benjamin, R. A., Benson, B. A., \& Cox, D. P. 2001, ApJ, 554, L225

Boulares, A., \& Cox, D. P. 1990, ApJ, 365, 544

Bregman, J. N. 1980, ApJ, 236, 577

Bregman, J. N. 2009, in The Role of Disk-Halo Interaction in Galaxy Evolution: Outflow vs. Infall?, arXiv:0907.3494v1

Breitschwerdt, D., \& Schmutzler, T. 1994, Nature, 371, 774

Breitschwerdt, D., \& Schmutzler, T. 1999, A\&A, 347, 650

Burrows, D. N., \& Mendenhall, J. A. 1991, Nature, 351, 629

Burrows, D. N., Singh, K. P., Nousek, J. A., Garmire, G. P., \& Good, J. 1993, ApJ, 406, 97

Carter, J. A., \& Sembay, S. 2008, A\&A, 489, 837

Chen, L.-W., Fabian, A. C., \& Gendreau, K. C. 1997, MNRAS, 285, 449

Crain, R. A., McCarthy, I. G., Frenk, C. S., Theuns, T., \& Schaye, J. 2010, MNRAS, 407, 1403

Cravens, T. E. 2000, ApJ, 532, L153

Cravens, T. E., Robertson, I. P., \& Snowden, S. L. 2001, J. Geophys. Res., 106, 24883 
de Avillez, M. A., \& Breitschwerdt, D. 2004, A\&A, 425, 899

Diehl, R., et al. 2006, Nature, 439, 45

Ferrière, K. 1995, ApJ, 441, 281

Ferrière, K. 1998, ApJ, 497, 759

Ferrière, K. M. 2001, Rev. Mod. Phys., 73, 1031

Fujimoto, R., et al. 2007, PASJ, 59, S133

Fujita, A., Martin, C. L., Mac Low, M.-M., New, K. C. B., \& Weaver, R. 2009, ApJ, 698, 693

Galeazzi, M., Gupta, A., Covey, K., \& Ursino, E. 2007, ApJ, 658, 1081

Gupta, A., Galeazzi, M., Koutroumpa, D., Smith, R., \& Lallement, R. 2009, ApJ, 707,644

Hasinger, G., Burg, R., Giacconi, R., Hartner, G., Schmidt, M., Trümper, J., \& Zamorani, G. 1993, A\&A, 275, 1

Henley, D. B., \& Shelton, R. L. 2008, ApJ, 676, 335

Henley, D. B., \& Shelton, R. L. 2009, ApJ, 701, 1880

Henley, D. B., \& Shelton, R. L. 2010, ApJS, 187, 388 (Paper I)

Joung, M. K. R., \& Mac Low, M.-M. 2006, ApJ, 653, 1266

Kalberla, P. M. W., Burton, W. B., Hartmann, D., Arnal, E. M., Bajaja, E., Morras, R., \& Pöppel, W. G. L. 2005, A\&A, 440, 775

Koutroumpa, D., Acero, F., Lallement, R., Ballet, J., \& Kharchenko, V. 2007, A\&A, 475, 901

Kuntz, K. D., \& Snowden, S. L. 2000, ApJ, 543, 195

Kuntz, K. D., \& Snowden, S. L. 2008, A\&A, 478, 575

Lei, S., Shelton, R. L., \& Henley, D. B. 2009, ApJ, 699, 1891

Marshall, F. J., Boldt, E. A., Holt, S. S., Miller, R. B., Mushotzky, R. F., Rose, L. A., Rothschild, R. E., \& Serlemitsos, P. J. 1980, ApJ, 235, 4

Masai, K. 1994, ApJ, 437, 770

Moretti, A., Campana, S., Lazzati, D., \& Tagliaferri, G. 2003, ApJ, 588, 696

Norman, C. A., \& Ikeuchi, S. 1989, ApJ, 345, 372

Pietz, J., Kerp, J., Kalberla, P. M. W., Burton, W. B., Hartmann, D., \& Mebold, U. 1998, A\&A, 332, 55

R Development Core Team 2008, R: A Language and Environment for Statistical Computing (Vienna: R Foundation for Statistical Computing)

Rasmussen, J., Sommer-Larsen, J., Pedersen, K., Toft, S., Benson, A., Bower, R. G., \& Grove, L. F. 2009, ApJ, 697, 79

Raymond, J. C., \& Smith, B. W. 1977, ApJS, 35, 419

Sanders, W. T., Kraushaar, W. L., Nousek, J. A., \& Fried, P. M. 1977, ApJ, 217, L87
Shapiro, P. R., \& Field, G. B. 1976, ApJ, 205, 762

Shelton, R. L. 1998, ApJ, 504, 785

Shelton, R. L. 1999, ApJ, 521, 217

Shelton, R. L. 2006, ApJ, 638, 206

Shelton, R. L., Sallmen, S. M., \& Jenkins, E. B. 2007, ApJ, 659, 365

Smith, R. K., et al. 2007, PASJ, 59, S141

Snowden, S. L., Burrows, D. N., Sanders, W. T., Aschenbach, B., \& Pfeffermann, E. 1995, ApJ, 439, 399

Snowden, S. L., Collier, M. R., \& Kuntz, K. D. 2004, ApJ, 610, 1182

Snowden, S. L., Cox, D. P., McCammon, D., \& Sanders, W. T. 1990, ApJ, 354, 211

Snowden, S. L., Egger, R., Finkbeiner, D. P., Freyberg, M. J., \& Plucinsky, P. P. 1998, ApJ, 493, 715

Snowden, S. L., Freyberg, M. J., Kuntz, K. D., \& Sanders, W. T. 2000, ApJS, 128,171

Snowden, S. L., \& Kuntz, K. D. 2007, Cookbook for Analysis Procedures for XMM-Newton EPIC MOS Observations of Extended Objects and the Diffuse Background, version 2.0 (ftp://legacy.gsfc.nasa.gov/xmm/software/ xmm-esas/xmm-esas-v2/xmm-esas.pdf)

Snowden, S. L., \& Kuntz, K. D. 2010, Cookbook for Analysis Procedures for XMM-Newton EPIC MOS Observations of Extended Objects and the Diffuse Background, version 4.0 (ftp://legacy.gsfc.nasa.gov/xmm/software/ xmm-esas/xmm-esas.pdf)

Snowden, S. L., Mebold, U., Hirth, W., Herbstmeier, U., \& Schmitt, J. H. M. M. 1991, Science, 252, 1529

Sommer-Larsen, J. 2006, ApJ, 644, L1

Strüder, L., et al. 2001, A\&A, 365, L18

Toft, S., Rasmussen, J., Sommer-Larsen, J., \& Pedersen, K. 2002, MNRAS, 335,799

Turner, M. J. L., et al. 2001, A\&A, 365, L27

Wang, Q. D. 1998, in Lecture Notes in Physics 506, The Local Bubble and Beyond, ed. D. Breitschwerdt, M. J. Freyberg, \& J. Trümper (New York: Springer), 503

Wilms, J., Allen, A., \& McCray, R. 2000, ApJ, 542, 914

Yan, M., Sadeghpour, H. R., \& Dalgarno, A. 1998, ApJ, 496, 1044

Yao, Y., \& Wang, Q. D. 2005, ApJ, 624, 751

Yao, Y., \& Wang, Q. D. 2007, ApJ, 658, 1088

Yao, Y., Wang, Q. D., Hagihara, T., Mitsuda, K., McCammon, D., \& Yamasaki, N. Y. 2009, ApJ, 690, 143

Yoshino, T., et al. 2009, PASJ, 61, 805 